\title{
Exploiting Compression Opportunities to Improve SpMxV Performance on Shared Memory Systems
}

\author{
KORNILIOS KOURTIS, GEORGIOS GOUMAS, and NECTARIOS KOZIRIS \\ National Technical University of Athens
}

\begin{abstract}
The Sparse Matrix-Vector Multiplication (SpMxV) kernel exhibits poor scaling on shared memory systems, due to the streaming nature of its data access pattern. To decrease memory contention and improve kernel performance we propose two compression schemes: CSR-DU, that targets the reduction of the matrix structural data by applying coarse-grained delta-encoding, and CSR-VI, that targets the reduction of the values using indirect indexing, applicable to matrices with a small number of unique values. Thorough experimental evaluation of the proposed methods and their combination, on two modern shared memory systems, demonstrated that they can significantly improve multithreaded $\mathrm{SpMxV}$ performance upon standard and state-of-the-art approaches.
\end{abstract}

Categories and Subject Descriptors: E.4 [Data]: Coding and Information Theory-Data compaction and compression; C.1.2 [Processor Architectures]: Multiple Data Stream Architectures (Multiprocessors); G.4 [Mathematics of Computing]: Mathematical Software

General Terms: Experimentation, Performance, Measurement

Additional Key Words and Phrases: Sparse matrix, data compression, memory bandwidth, shared memory systems

The work presented in this article was supported by the 03ED255 research project, implemented within the framework of the "Reinforcement Programme of Human Research Manpower" (PENED) and cofinanced by National and Community Funds (20\% from the Greek Ministry of DevelopmentGeneral Secretariat of Research and Technology and 80\% from B.D.-European Social Fund).

This article extends the papers "Optimizing Sparse Matrix Vector Multiplication Using Index and Value Compression" published in Proceedings of the 2008 Conference on Computing Frontiers (CF"08), and "Improving the Performance of Multithreaded Sparse Matrix-Vector Multiplication Using Index and Value Compression" published in Proceedings of the 37th International Conference on Parallel Processing, 2008 (ICPP'08). The main additions with regard to prior work presented in this article are: (a) Extension of the CSR-DU method to support units with contiguous nonzero elements; (b) development and evaluation of a new method that combines CSR-DU and CSR-VI; (c) evaluation of all proposed methods in a new-generation system, that implements NUMA; (d) comparison with state-of-the-art BCSR storage format.

Authors' address: K. Kourtis, G. Goumas, and N. Koziris, School of Electrical and Computer Engineering, Computing Science Laboratory, National Technical University of Athens, Athens, Greece; email: \{kkourt, goumas, nkoziris\}@cslab.ece.ntua.gr.

Permission to make digital or hard copies of part or all of this work for personal or classroom use is granted without fee provided that copies are not made or distributed for profit or commercial advantage and that copies show this notice on the first page or initial screen of a display along with the full citation. Copyrights for components of this work owned by others than ACM must be honored. Abstracting with credit is permitted. To copy otherwise, to republish, to post on servers, to redistribute to lists, or to use any component of this work in other works requires prior specific permission and/or a fee. Permissions may be requested from Publications Dept., ACM, Inc., 2 Penn Plaza, Suite 701, New York, NY 10121-0701 USA, fax +1 (212) 869-0481, or permissions@acm.org. (C) 2010 ACM 1544-3566/2010/12-ART16 $\$ 10.00$

DOI 10.1145/1880037.1880041 http://doi.acm.org/10.1145/1880037.1880041

ACM Transactions on Architecture and Code Optimization, Vol. 7, No. 3, Article 16, Pub. date: December 2010. 
ACM Reference Format:

Kourtis, K., Goumas, G., and Koziris, N. 2010. Exploiting compression opportunities to improve SpMxV performance on shared memory systems. ACM Trans. Architec. Code Optim. 7, 3, Article 16 (December 2010), 31 pages.

DOI $=10.1145 / 1880037.1880041 \mathrm{http} / / /$ doi.acm.org/10.1145/1880037.1880041

\section{INTRODUCTION}

The ineffectiveness of traditional techniques, like frequency scaling and ILP exploitation, for enhancing processor performance led to a technology shift towards chip multiprocessor (CMP or multicore) designs, both in terms of commodity products and future research directions [Hennessy and Patterson 2007; Geer 2005]. As a result, a large number of research efforts target the scalability challenges that arise in applications when a large number of processing units operate on a shared memory hierarchy.

The scalability behavior of an application in a shared memory environment depends on its data access pattern. Applications with no data dependencies and good temporal locality scale well: each core can work independently using local data residing in its cache, without interfering with the operation of other cores. On the contrary, applications with streaming access patterns tend to exhibit poor scaling, due to contention on the memory subsystem.

An important and ubiquitous computational kernel with streaming memory access pattern is the Sparse Matrix-Vector multiplication $(\mathrm{SpMxV})$. $\mathrm{SpMxV}$ is used in a large variety of applications in scientific computing and engineering. For example, it is the basic operation of iterative solvers, such as Conjugate Gradient (CG) and Generalized Minimum Residual (GMRES). CG and GMRES are extensively used to solve sparse linear systems resulting from the simulation of physical processes described by partial differential equations [Saad 2003]. Furthermore, $\mathrm{SpMxV}$ is a member of one of the "seven dwarfs," which are classes of applications that are believed to be important for at least the next decade [Asanovic et al. 2006].

The distinguishing characteristic of sparse matrices is that they are populated by a large number of zero elements, making it highly inefficient to perform operations using typical (dense) array structures. Special storage schemes are used instead, which target both the sparing storage of the matrix in terms of space, and the efficient execution of various operations by performing only the necessary actions. The common approach is to store only the nonzero values of the matrix, and employ additional indexing information about the position of these values. In this article a distinction will be made between data representing matrix structure (index data), and data representing numerical values (value data).

Our recent work [Goumas et al. 2008], as well as related literature [Anderson et al. 1999], has identified the memory subsystem as the main performance bottleneck of the $\mathrm{SpMxV}$ kernel when executed in a uniprocessor environment. Obviously, if more processing units access the main memory, this bottleneck will become more severe. Consequently, it is expected that a multithreaded version of the kernel, targeted for shared memory architectures, will have poor 
performance scaling as the number of utilized cores increases. An approach for alleviating this problem to reduce the data accessed during the execution of the kernel (working set).

In this direction and using the standard CSR [Barrett et al. 1994; Saad 2003] sparse matrix storage format as a starting point, we propose two new formats: CSR-DU and CSR-VI [Kourtis et al. 2008a]. CSR-DU is a general format that reduces index data volume using coarse-grained delta-encoding for the compression of column indices. CSR-VI is a specialized format that exploits the data redundancy of matrices with a large number of common values using indirect value accesses. We also consider the combination of these two formats (CSR-DUVI), where both indices and values are compressed. The intrinsic basis of compression is to trade data storage volume for computation overhead. We argue that as the number of processing cores that share the memory subsystem increases, this tradeoff will become more beneficial for the performance of memory bound applications such as $\mathrm{SpMxV}$, even if it results in degraded performance in the uniprocessor case.

To evaluate our proposed methods we perform experimental tests over a large number of matrices. We focus our attention on the scalability issues that arise as core count increases. Our experimental platform consists of two systems that correspond to the ends of the commodity hardware spectrum regarding memory performance: an SMP system with centralized memory, and a new generation NUMA system, with a strong architectural focus on memory throughput performance. Our experimental results confirm that the multithreaded version of the $\mathrm{SpMxV}$ kernel exhibits poor scalability. We compare CSR-DU against CSR and BCSR [Im and Yelick 2001], which constitutes the most popular optimized format. CSR-DU significantly outperforms CSR and BCSR when all available cores participate in the computation. CSR-VI is able to drastically reduce the working set in a large subset of our experimental matrix suite and considerably improve performance. Finally, experimental results for CSR-DUVI demonstrate the effectiveness of the combination of index and value compression.

The rest of the article is organized as follows: Section 2 sets the context, by providing an introduction to issues related to this work. Sections 3 and 4 present the proposed compression methods, and Section 5 discusses the experimental evaluation results. Section 6 discusses the related literature, and Section 7 concludes the article.

\section{PRELIMINARIES}

\subsection{Shared Memory Architectures}

Shared memory architectures with multiple processors (SMPs) have been studied extensively in the past [Culler and Singh 1999]. The current trend of multicore processors, along with indications for many-core next-generation processors [Hsu et al. 2005], has motivated the research community to revisit the performance issues of shared memory architectures and investigate methods for scaling applications up to a large number of processing units. The major 


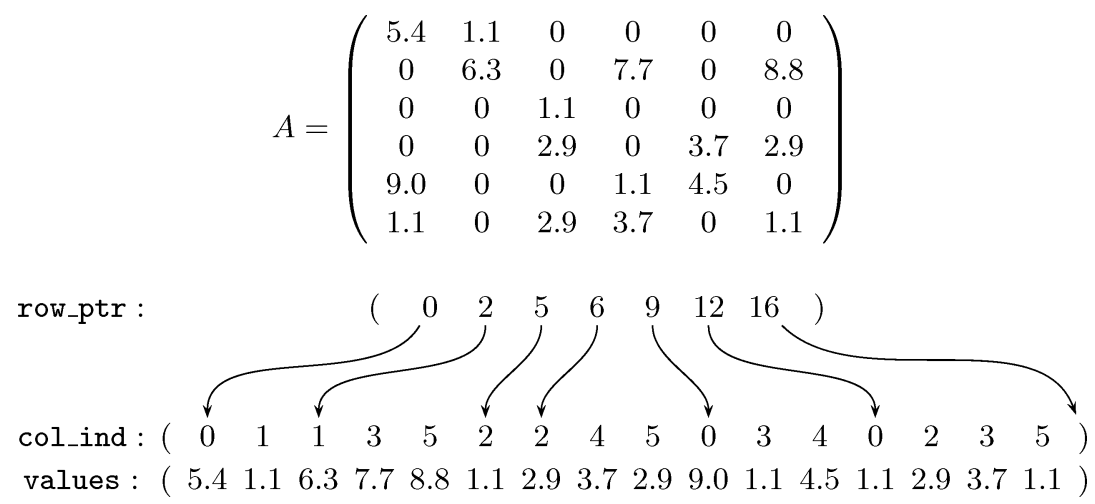

Fig. 1. Example of the CSR storage format.

performance problem of these architectures is the main memory, which is typically centralized and shared among all processors.

It is worth mentioning that a difference between multicore processors and classic SMP systems is that in the former different cores may share a part of the cache hierarchy (e.g., the L2 or the L3 cache), instead of just the main memory. Cache sharing is an important factor of the system's performance and can be either constructive or destructive, depending on the application and on whether threads scheduled on the cores that share a cache operate on common data or not.

As core count increases, CPU designers turn to more scalable designs like Non-Uniform Memory Access (NUMA) architectures where the memory is distributed on different nodes, connected via a scalable interconnect. For a given CPU, NUMA memory nodes are characterized as either local, or remote. Accessing a local node is faster than accessing a remote node. This architecture mitigates the memory bandwidth bottleneck, since it allows different CPUs to operate on different NUMA nodes. In general, these systems provide a coherent unified view of memory, and is up to the operating system or the programmer to distribute data in different nodes to maximize performance.

\subsection{Sparse Matrix Formats and the SpMxV Operation}

The most commonly used storage format for sparse matrices is the Compressed Sparse Row (CSR) format [Barrett et al. 1994; Saad 2003]. In CSR the matrix is stored in three arrays: values, row_ptr and col_ind. The values array stores the nonzero elements of the matrix in row-major order, while the other two arrays store indexing information: row_ptr contains the location of the first (nonzero) element of each row within the values array, and col_ind contains the column number for each nonzero element. An example of the CSR format for a $6 \times 6$ sparse matrix is presented in Figure 1 . The size of the values and col_ind arrays are equal to the number of nonzero elements ( $n n z)$, while the row_ptr array size is equal to the number of rows (nrows) plus one. Other generic formats for sparse matrices are the Compressed Sparse Column (CSC), which is similar to CSR storing columns instead of rows, and the Coordinate 
format ( $\mathrm{COO})$, where each nonzero is stored as a triplet along with matrix location coordinates.

The $\mathrm{SpMxV}$ operation $(y=A x)$, is the multiplication of a sparse matrix $A$ and a (dense) vector $x$, with the result stored in another (dense) vector $y$. The CSR implementation for a matrix with $N$ rows is:

$$
\begin{aligned}
& \text { for }(i=0 ; i<N ; i++) \\
& \quad \text { for }(j=\text { row_ptr[i]; j<row_ptr }[i+1] ; j++) \\
& \quad y[i]+=\operatorname{values}[j] * x[\operatorname{col} \text { _ind }[j]] ;
\end{aligned}
$$

The working set $(w s)$ consists of the matrix and vector data. Its size is expressed by the following formula where $i d x \_s$ and $v a l \_s$ represent the storage size required for an index and a value respectively.

$$
w s=\overbrace{\left(n n z \cdot\left(i d x \_s+v a l \_s\right)+(\text { nrows }+1) \cdot i d x \_s\right)}^{\text {sparse matrix }}+\overbrace{(\text { nrows }+ \text { ncols }) \cdot v a l_{\_} s}^{\text {vectors }} .
$$

Because the majority of real-life sparse matrices satisfy: $n n z \gg$ nrows, ncols, we can approximate the above expression by $w s=n n z \cdot\left(i d x \_s+v a l \_s\right)$. Commonly, a 4-byte integer is used for index storage, due to memory size restrictions that limit $x$ and $y$ vectors to a maximum of $2^{32}$ elements. Numerical data, however, normally require double precision. Under these assumptions, that is, $i d x_{s} s=4$ and $v a l \_s=8$, value data constitute the larger portion of the working set by a factor of $2 / 3$. For this reason, value data compression is expected to have a greater impact to overall working set reduction.

The 2/3 factor is a result of memory size limitations (we assume that the sparse matrix resides in memory) and can change in the future. Specifically, matrices with dimensions larger than $2^{32}$, require indices larger than 32 bits. We consider a square sparse matrix with $n=$ nrows $=n c o l s=2^{32}$ and $n n z=$ $100 \cdot n=100 \cdot 2^{321}$. The required CSR storage would be $100 \cdot 2^{32} \cdot(4+8)$ bytes $\approx 4.7 \mathrm{TiB}$. Currently, only some high-end enterprise servers contain this much memory. However, given the current rate of advancement, it is probable that near-future commodity hardware will support these capabilities.

\subsection{Multithreaded SpMxV}

There are several data partitioning schemes for parallelizing the $\mathrm{SpMxV}$ kernel on a shared memory architecture. For CSR, coarse-grained row partitioning is usually applied [Williams et al. 2007] where different blocks of rows are assigned to different threads (see Figure 2). Threads operate on disjoint subsets of row_ptr, col_ind, values, and y arrays. The only sharing occurs in $\mathrm{x}$ array data, but it does not constitute a performance problem. Access to $\mathrm{x}$ is read only, allowing efficient data caching over all processors. One could argue that the common use of $\mathrm{x}$ offers potential for constructive cache sharing. In practice, however, this potential is not realized, because shared data constitute a small part of the working set and cache space is limited.

${ }^{1}$ The number 100 has been chosen because it is close to the average value of $n n z / n$ for our matrix suite (see Table II) 

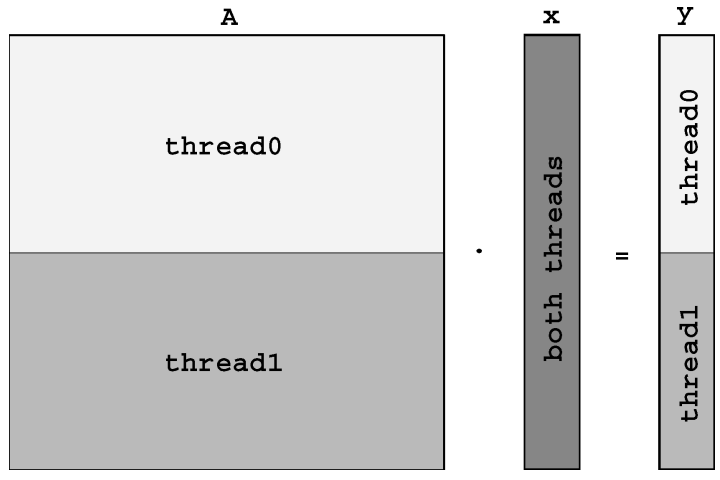

Fig. 2. Row partitioning on $\mathrm{SpMxVfor} \mathrm{two} \mathrm{threads.}$

The complementary approach to row partitioning is column partitioning where each thread is assigned a block of columns. Although this approach is more naturally applied to the CSC format, it can also be applied to CSR. An advantage of column partitioning is that each thread operates on a different part of the $\mathrm{x}$ vector, which leads to better temporal locality for its elements in case of distinct caches. A disadvantage, however, is the possibility of cache-line ping-pongs, since each thread performs updates over all y elements. Having each thread use its own y array eliminates this problem. The final result can be obtained by adding the partial y arrays. Additionally, applying column partitioning to CSR may result in performance degradation, due to loop overheads caused by empty or small rows.

Block partitioning is the combination of the two aforementioned schemes where each thread is assigned an arbitrary two-dimensional block. It has the benefit of allowing configurable data sizes for each thread. For this reason, it is applied when the available memory space is limited (e.g., in the Cell processor [Gschwind et al. 2006]). Although we consider only row partitioning in our experimental evaluation, we argue that the proposed formats will result in similar performance improvements for other partitioning schemes as well.

Another issue of $\mathrm{SpMxV}$ parallelization is balancing each thread's workload. We apply a static balancing scheme based on the non-zero elements. Each thread is assigned approximately the same number of elements and thus the same number of floating-point operations.

\section{INDEX COMPRESSION}

\subsection{Motivation and General Approach}

Sparse storage formats traditionally try to exploit contiguous elements, either in one (Figure 3(a)) or two dimensions (Figure 3(b)). Examples include the BCSR format [Saad 1994; Im and Yelick 2001], and the variable-length onedimensional block format described in Pinar and Heath [1999]. BCSR can be viewed as a generalization of CSR where the granularity unit is an $r \times c$ dense block. The effect to overall matrix size when converting from CSR to BCSR depends on the aptitude of the selected block shape to capture the matrix 


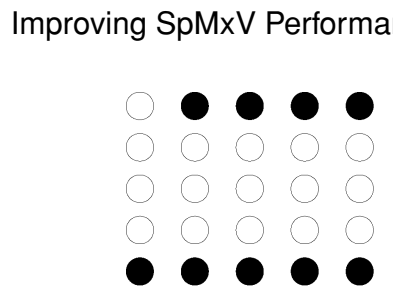

(a)

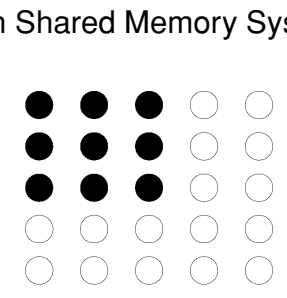

(b)

Fig. 3. Sparse matrix patterns: (a) sequential elements, (b) two-dimensional blocks.

structure. If resulting blocks contain a small number of zeroes, significant index reduction is achieved. For example, perfect blocking - that is, none of the BCSR blocks contain zeroes-leads to an index reduction by a factor of $r \cdot c$. On the contrary, zeroes included in BCSR blocks must be explicitly added to value data, because all BCSR blocks are stored in a dense form. This, depending on the matrix structure and selected block shape, may lead to overall matrix size increase.

Our approach is based on the general premise that sparse matrices have dense areas that do not necessarily contain contiguous nonzero elements (i.e., areas where elements are close but not sequential). These areas can contribute significantly to index data size reduction when delta encoding is used to reveal the highly redundant nature of the col_ind array [Willcock and Lumsdaine 2006]. In a delta encoding scheme the column indices are replaced with deltas, each of which is defined as the difference of the current index with the previous one. Since delta values are positive and less or equal than their corresponding column indices, they can be stored in smaller size integers, leading to index data size reduction.

Instead of encoding each delta value to use only the necessary number of bytes, we propose a coarse-grained approach where the matrix is divided into units with a variable number of elements. For each of these units, the maximum delta value is calculated, and a size that can represent this value is selected for all the delta values of the unit. This technique enables for innermost loops with minimum overheads by sacrificing some space. Normally, if each delta value was encoded separately, the innermost loop of the $\mathrm{SpMxV}$ kernel would contain branches to implement decoding. Misprediction of these branches in execution time leads to significant performance degradation.

An important factor for the performance of this method lies in the choice of the unit size. If the size is too small, the decompression overhead introduced will dominate the performance gain from the compression. On the contrary, if the unit size is large, there will be less opportunities for compression, because a single large delta value will enforce big storage requirements for the whole unit.

This approach demonstrates a more abstract optimization strategy for the $\mathrm{SpMxV}$ kernel, used to exploit matrix-specific structure information. To this direction the concept of units could be extended to support more types of regularities, thus providing a number of advantages: (a) It can be used to exploit local regularities in specific areas of the matrix, (b) It operates on a coarse-grained level and thus can effectively minimize the introduced overhead by selecting sufficiently large sizes and (c) it can bound the search space for regularities or 


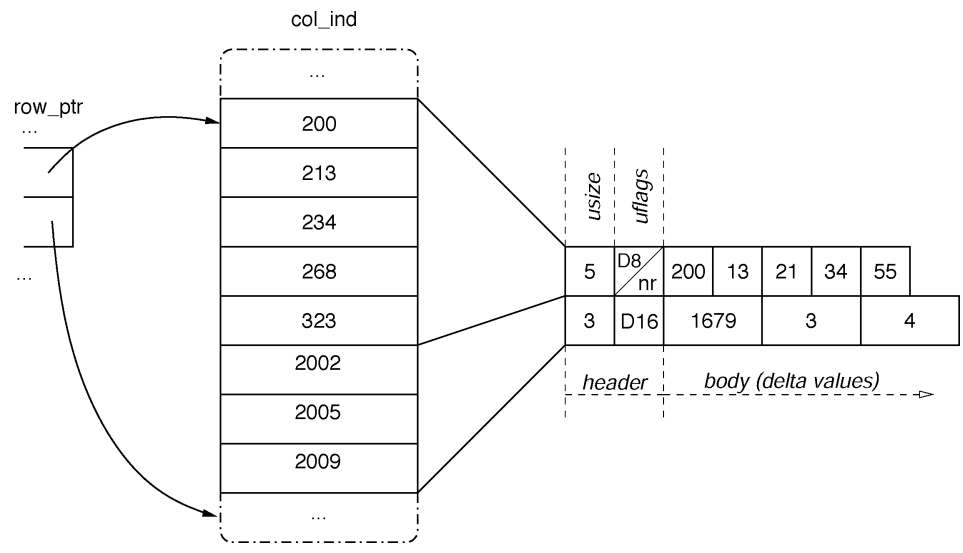

Fig. 4. Example of the CSR-DU storage format where a row is split into two units.

patterns and assure that the compression procedure will not exceed the available resources (e.g., time or storage). In Section 3.4 we discuss a method for exploiting sequential elements.

\subsection{The CSR-DU Storage Format}

The CSR-DU (CSR with Delta Units) storage format divides index data into units which are stored in a single byte-array called ctl. Each unit is limited to elements of a single row and consists of two parts. First, the header where the unit's properties are stored. Second, the main body where the delta-encoded column indices are stored. The header, in its simplest form, consists of two onebyte fields: (a) usize, the number of elements the unit contains and (b) uflags, a bit-vector encoding the unit's characteristics. Since usize is stored in a single byte, the maximum possible number of elements per unit is $2^{8}=256$. The size $(1,2,4 \text { or } 8 \text { bytes })^{2}$ of the delta values stored in the main body can be extracted from the uflags field, along with a marker that designates the beginning of a new row. Figure 4 presents an example of the CSR-DU format. In this example a row with 8 elements is split into two units. The first unit has 5 elements, 1-byte delta size, and a designator for a new row ( $\mathrm{nr}$ ). The second unit has 3 elements, and 2-byte delta size.

The compression procedure of CSR-DU is straightforward. It can be performed in $O(n n z)$ steps by scanning the matrix elements once, while keeping appropriate information in buffers until a unit is finalized. This means that the construction process of CSR-DU involves no overhead in terms of time complexity compared to CSR. An important decision during this procedure is when to finalize a unit. We implemented a simple approach where a unit is finalized if (a) a new row starts in the next element, or (b) the maximum unit size is reached. A more elaborate scheme would be to finalize a unit if a new element increases the delta storage size, as long as the unit already contains more than a predetermined number of elements.

28 bytes delta values are unnecessary due to hardware limitations, but supported for completeness. 


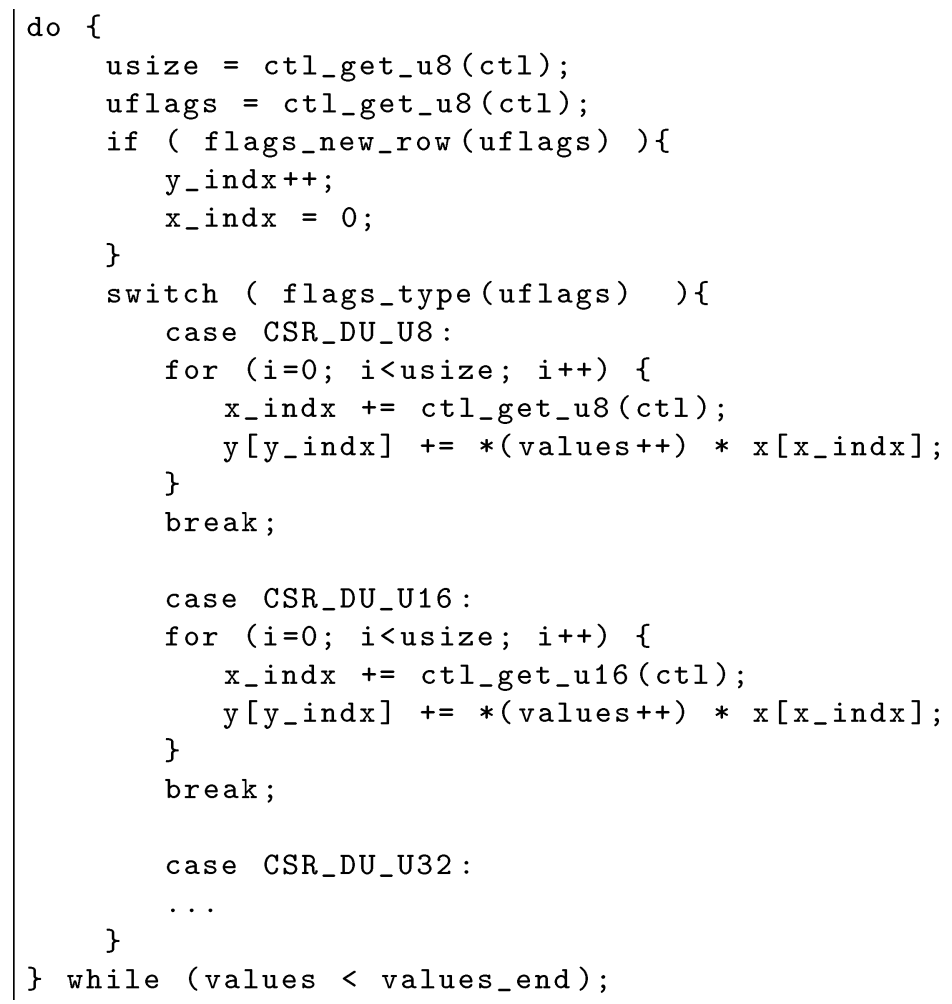

Fig. 5. SpMxV CSR-DU implementation.

The $\mathrm{SpMxV}$ implementation for the CSR-DU storage format is presented in Figure 5. Access to the ctl array is performed via macros (e.g., ctl_get_u16()) that return the appropriate value and advance the array pointer as necessary. Initially, the uflags and usize header fields are extracted from the ctl byte-array. If the unit belongs to a new row, appropriate initializations are performed: the $\mathrm{y}$ index is increased and the $\mathrm{x}$ index is zeroed. Finally, the appropriate multiplication code is executed based on the unit type. The innermost loops of the multiplication code for the various cases do not contain any branches, which allows for fast execution by the processor.

Parallelization is similar to CSR. For example, we consider the row partitioning scheme described in Section 2.3. Each thread needs an offset in the ctl, values, and y arrays to mark the beginning of its data, and the total number of rows that have been assigned to it. The next paragraphs discuss extensions to CSR-DU format for performance improvement.

\subsection{Unit Offsets}

A problem with CSR-DU, as described in the previous paragraphs, is that the unit's first delta value can be significantly larger than the rest, imposing an unnecessarily large storage size for the rest of the deltas. In the example of Figure 4 the density of the second unit's elements allows for one-byte delta 


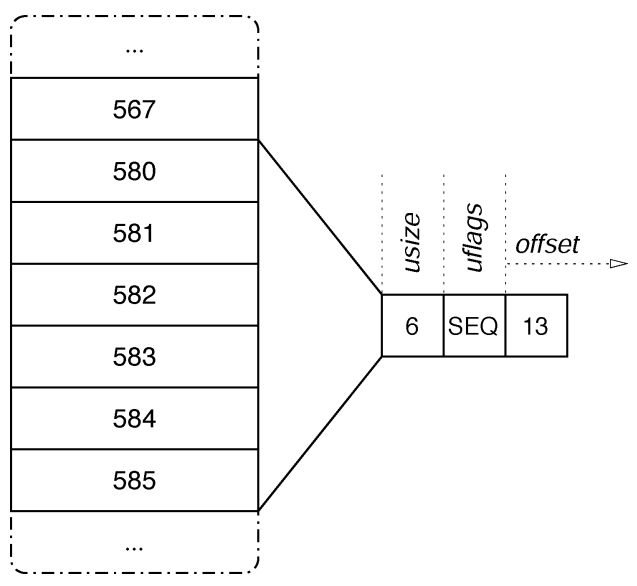

Fig. 6. Example of sequential elements unit.

values. However, the large distance from the first unit dictates two-byte storage. To counter this problem, we modify the original CSR-DU format to include a column index offset from the previous unit in the header. The offset is called ujmp and is stored as a (positive) variable-length integer at the end of the header. This technique improves compression of the column indices at no cost for performance since the change does not effect innermost loops. For the implementation of the variable-length integers, we used a simple scheme where the integer's bits in its normal form are divided in 7-bits parts. These parts are stored in consecutive bytes in which the MSB is used to mark the last byte of the integer.

\subsection{Sequential Elements}

Although delta encoding can significantly reduce index data volume, it does not handle the occurrence of sequential elements efficiently. If all unit elements are sequential column indexing information can be completely omitted. This, not only reduces the working set size, but also eliminates indirect accesses on $\mathrm{x}$ allowing for better optimization from both the compiler and the CPU. We extend CSR-DU, in a way similar to the format presented in Pinar and Heath [1999], to support units containing sequential elements. An example of this unit type (sequential units) is illustrated in Figure 6. Besides the usize and uflags fields, the unit data also contain the column index offset from the previous unit as a variable length integer. Note that if unit offsets are used, then the last field of the unit coincides with ujmp.

An important parameter that needs to be considered during the compression phase is the minimum possible size for the sequential units. We will refer to this parameter as seq. Consecutive elements of size less than seq will be encoded using delta encoding as described in previous sections. Tuning of this parameter prevents performance degradation from sequential units with small size. For example, if $s e q=1$ then all units of the matrix will be encoded as sequential. This will result in poor performance if the matrix does not contain enough sequential 
elements. In general, the effect of $s e q$ on $\mathrm{SpMxV}$ performance depends on: (a) the architecture of the execution platform and (b) the structure of the matrix (e.g., frequency of sequential units).

\subsection{Alignment of ctl Array Values}

Another issue with the CSR-DU format is that packing of delta values larger than 1 byte in the ctl array may lead to unaligned storage. For example in the case of Figure 4, if the first field of the ctl array is aligned, then the three 16-bit deltas in the second unit are stored in an unaligned manner. Some ISAs disallow unaligned access. Others (e.g., the x86 and x86_64 ISAs) include instructions that allow unaligned access, but may result in performance degradation. In our implementation, we pad the ucis sections in the ctl array, so that the accesses of delta indices are always performed in an aligned manner.

\section{VALUE COMPRESSION}

\subsection{Motivation and General Approach}

As mentioned in Section 2.2, values typically constitute the larger part of the working set of a CSR matrix because they require 64-bit storage. Hence, value compression is potentially more beneficial than index compression in terms of working set reduction. Conversely with index data, value data do not inherently contain redundancy in the general case. Moreover, the compression of floating point values is not as straightforward as integers, because floating point arithmetic operations produce rounded results.

Nevertheless, we have noticed that a significant number of matrices from our experimental set contain a small number of unique values, relative to the total nonzero values $(n n z)$. From an information theory perspective this results in low entropy for the value data, making them a good target for compression. Since we aim for a computationally light decompression scheme we follow a simple approach: instead of storing all the $n n z$ values, we store only the common values and pointers to them. If the total-to-unique values ratio is high enough, the working set data volume will be reduced. Adequate size reduction can lead to $\mathrm{SpMxV}$ execution time decrease, despite the overhead induced by indirectly accessing each value.

\subsection{The CSR-VI Storage Format}

The CSR-VI (CSR with Values Indirect) format replaces the CSR values array with two arrays: vals_unique and val_ind. The first contains only the unique matrix values. The second contains indices in the vals_unique array for each of the $n n z$ matrix elements. To achieve working set size reduction, val_ind size must be significantly smaller than values size. A simple approach towards this goal is to reduce the storage requirements of individual value indices compared to the storage requirements of original values. Hence, in CSR-VI the value index size is determined by the number of the unique values that need to be addressed. For example, if there exist $u v$ unique values and $2^{8}<u v \leq 2^{16}$, then a 2-byte integer will be used for each value index. Although this approach 


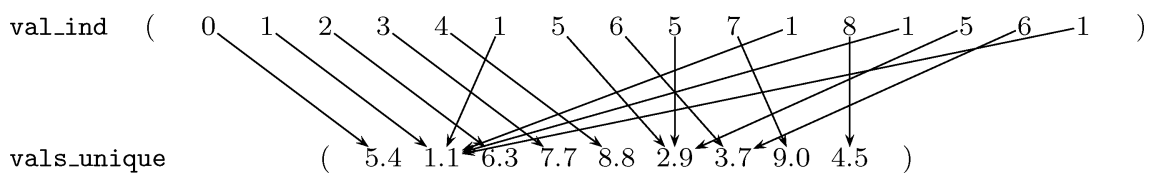

Fig. 7. Example of the value indexing structure for the CSR-VI format for the matrix presented in Figure 1.

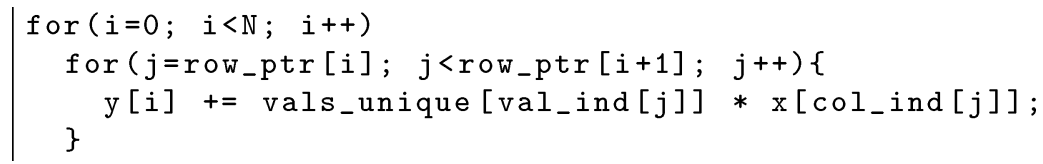

Fig. 8. SpMxV kernel for the CSR-VI storage format.

does not optimally compresses value indices, it induces only a small overhead because it does not add any branches. An example of this value structure is presented in Figure 7, which contains the matrix values from Figure 1.

The SpMxV kernel implementation for CSR-VI is presented in Figure 8. It can be easily derived from the CSR case by replacing the access of values with an indirect access of vals_unique based on the value of val_ind. Even though the resulting code contains an additional memory reference for each of the $n n z$ elements, it will lead to fewer data being transferred from memory when the number of unique values is relatively small. Nevertheless, working set reduction alone is not sufficient to achieve performance improvement; the additional overhead of indirect accesses must be amortized. The compression for CSR-VI is implemented using a hash table and, as in CSR-DU, its complexity is $O(n n z)$.

\subsection{Combining CSR-VI and CSR-DU}

CSR-DU and CSR-VI can be applied independently, because they operate on different data sets: CSR-DU is concerned with index data, while CSR-VI with matrix numerical values. We will refer to their combination as CSR-DUVI, a storage format that applies compression to both index and value data. Obviously, CSR-DUVI is not a general format, but can only be applied to matrices with a small number of unique values. A part of the CSR-DUVI SpMxV kernel implementation is shown in Figure 9.

\section{EXPERIMENTAL EVALUATION}

\subsection{Experimental Setup}

We conduct experiments on two systems. The first system is equipped with two quad-core Intel Harpertown processors (Figure 10). Cores operate at $2 \mathrm{GHz}$, include two private L1 $32 \mathrm{KiB}$ caches (instructions and data), and are grouped in pairs that share a unified $6 \mathrm{MiB}$ L2 cache. The processors interface with main memory via the Intel 5000p Memory Controller $\mathrm{Hub}(\mathrm{MCH})$ which provides four channels of fully buffered DDR2 DIMM (FB-DDR2) memory. 


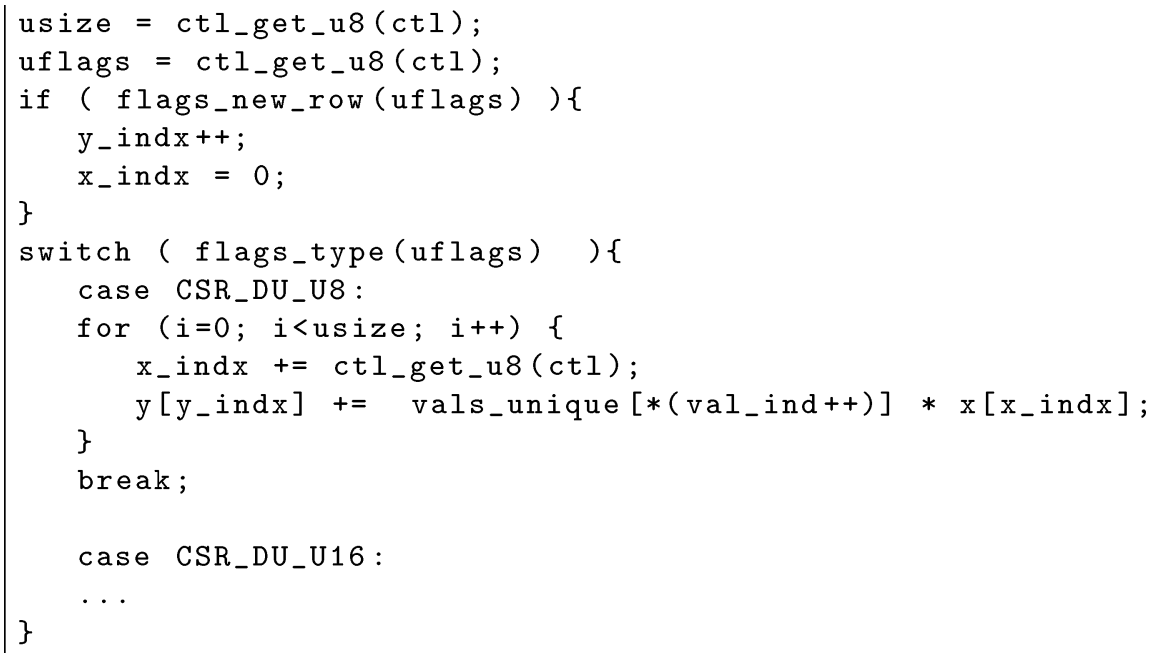

Fig. 9. Code snippet for the $\mathrm{SpMxV}$ kernel for CSR-DUVI.

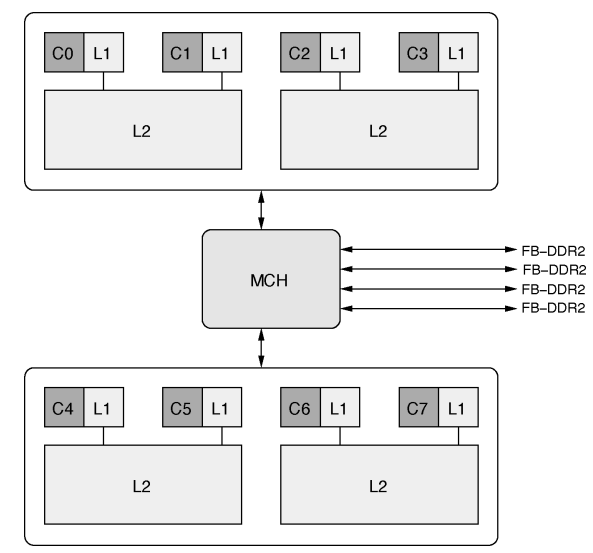

Fig. 10. An 8-core system comprising two Harpertown processors.

In contrast with Harpertown, which uses a unique interface with main memory, the second system consists of two Intel Nehalem ${ }^{3}$ processors that implement NUMA. Each processor has four cores that operate on $2.8 \mathrm{GHz}$. Each core has private $\mathrm{L} 1$ (32KiB instructions and data) and L2 (256KiB unified) caches. Cores of the same processor share an L3 (8MiB unified) cache. Moreover, Nehalem is equipped with an on-chip memory controller that supports three DDR3 memory channels. Communication with other memory nodes and I/O devices is implemented via QuickPath (QP) interconnect point-to-point links (Figure 11). Additionally, Nehalem cores implement Simultaneous MultiThreading (SMT), providing two different thread contexts per core.

As depicted in Figures 11 and 10, real-world systems usually employ a hierarchical topology where different core sets share different parts of the memory

${ }^{3} \mathrm{An}$ initial performance evaluation of a Nehalem system can be found in Barker et al. [2008].

ACM Transactions on Architecture and Code Optimization, Vol. 7, No. 3, Article 16, Pub. date: December 2010. 


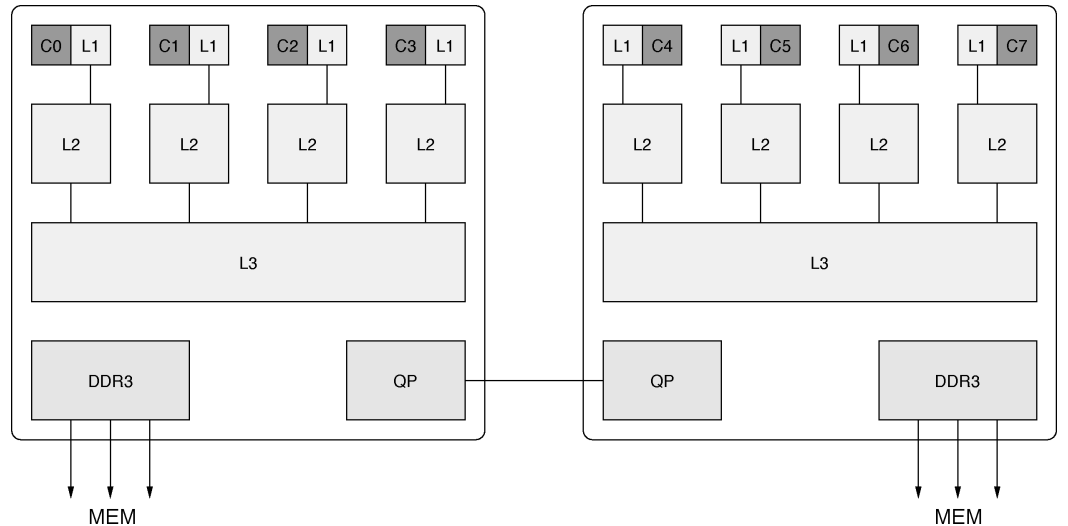

Fig. 11. An 8-core system comprising two Nehalem processors.

Table I. Overview of the Systems Used in the Experimental Evaluation

\begin{tabular}{|l|c|c|}
\hline System & Harpertown & Nehalem \\
\hline Model & E5405 & X5560 \\
Frequency (Ghz) & 2.0 & 2.8 \\
L1 (data/instruction) & $32 \mathrm{k} / 32 \mathrm{k}$ & $32 \mathrm{k} / 32 \mathrm{k}$ \\
L2 (unified) & $6 \mathrm{M}(1 / 2$ cores $)$ & $256 \mathrm{k}(1 /$ core $)$ \\
L3 (unified) & - & $8 \mathrm{M}(1 /$ chip) \\
Multithreading configuration & $2 \mathrm{c} 0 \times 2 \mathrm{c} 1 \times 2 \mathrm{~d}$ & $2 \mathrm{t} \times 4 \mathrm{c} \times 2 \mathrm{~d}$ \\
\hline
\end{tabular}

hierarchy. To distinguish between different scheduling configurations we will use a notation that explicitly describes the number of threads used in each level of the hierarchy. The levels are represented as:

$t$ : SMT threads on the same core (Nehalem).

c0 : cores that share L2 (Harpertown)

c1 : cores that do not share L2 (Harpertown)

c : cores that do not share L3 (Nehalem)

d : different dies (Harpertown and Nehalem)

Table I provides a concise overview of the two processors used for our experimental evaluation.

For our evaluation we compiled our code with gec 4.3.2, and performed our experiments in a 64-bit version of the Linux operating system (2.6.30). We explicitly parallelized all versions of the $\mathrm{SpMxV}$ kernel using the pthreads interface of the GNU libc library (NPTL 2.7). Moreover, we bound threads to specific cores using the sched_setaffinity() system call, and we allocated memory from specific NUMA nodes using the libnuma library (version 2.0.2).

We set the default storage size for indices and values to 32 and 64 bits respectively. Furthermore, we optimized the $\mathrm{SpMxV}$ code to write the y[i] value at the end of each innermost loop, by keeping the intermediate result in a register. The experiments were conducted by measuring the execution time of 128 consecutive $\mathrm{SpMxV}$ operations. We made no attempt to artificially pollute 


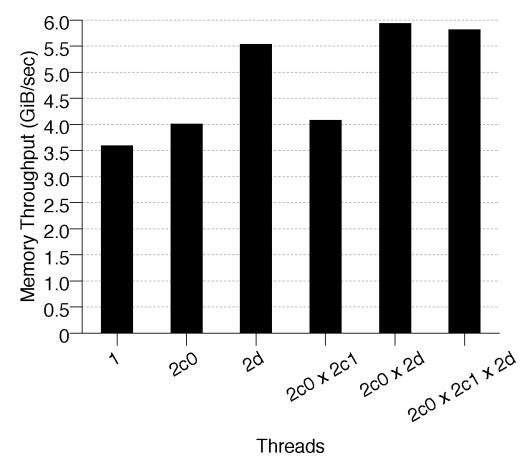

Fig. 12. Harpertown read memory throughput for different thread configurations.

the cache after each iteration, to better simulate iterative scientific application behavior where matrix data are present in the cache hierarchy, because either they have just been produced, or they were recently accessed. Additionally, we set $x$ to be the $y$ vector of the previous iteration, so that our benchmark has similar behavior to scientific methods based on $\mathrm{SpMxV}$ (e.g., GMRES). Setting $y$ as $x$, however, restricts our matrix suite to contain only square matrices. Finally, we applied the row partitioning scheme to all multithreaded implementations.

\subsection{Memory Throughput Benchmark}

To quantify system limits and the role of the various micro-architectural characteristics, we developed a benchmark to measure maximum throughput when different threads read data from the main memory. These measurements can be used to reveal system performance trends for applications with intensive streaming reads, such as the $\mathrm{SpMxV}$ kernel. The benchmark allocates and initializes large memory areas and subsequently performs read operations using streaming instructions (x86 Streaming SIMD Extensions - SSE).

Results for the Harpertown system are shown in Figure 12, which illustrates the achieved memory throughput for different scheduling configurations. As expected, scalability is poor. For example, when all available cores are used, the memory throughput is increased by a factor of 1.62 relevant to the single thread scenario. This scalability problem is more intense for threads that operate on the same die: two threads in the same core achieve only a 1.12 throughput increase when compared to the serial case, while the same number for threads in different dies achieve about 1.54. Another observation from the diagram is that memory accesses from different threads may lead to performance degradation due to contention. For example, the throughput of 8 threads is less than the throughput of the $2 \mathrm{c} 0 \times 2 \mathrm{~d}$ configuration.

The results for the Nehalem system are presented in Figure 13. Figure 13(a) shows the achieved memory throughput of one thread for different NUMA memory allocation policies. Local node allocation outperforms remote and interleaved policy by a significant factor (1.51 and 1.25, respectively). Figure 13(b) presents results for various thread configurations when using memory allocated on the local NUMA node for each thread. A single thread achieves 


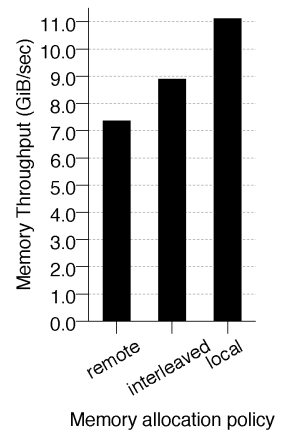

(a)

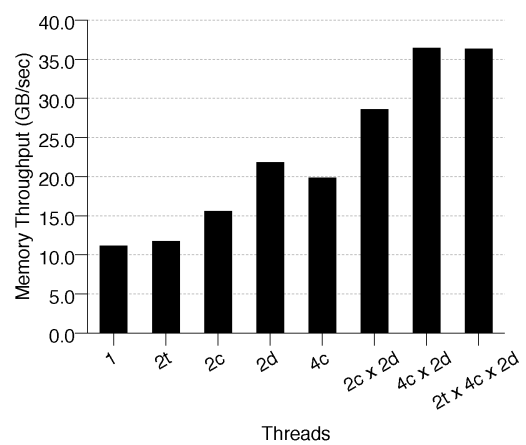

(b)

Fig. 13. Read memory throughput for the Nehalem architecture: (a): Nehalem read memory throughput for one thread and different NUMA allocation policies. (b): Nehalem read memory throughput for different thread configurations. Local node allocation policy is used.

$11.1 \mathrm{GiB} / \mathrm{sec}$ when reading from a local node, which constitutes a 3.1 improvement over Harpertown single-thread performance. Moreover, NUMA allows for good scalability when different processors are used. The speedup achieved for two threads running on different dies is, as expected, almost linear (1.96), and when all cores are utilized the speedup is 3.27. It also is worth noting that when all cores are utilized the Nehalem processor outperforms the Harpertown processor by a factor of $6.25(36.4 \mathrm{vs} 5.9 \mathrm{GiB} / \mathrm{sec})$ in this benchmark.

A comparison between these two systems shows a technology shift towards designs that focus on memory throughput performance, and indicates the importance of the memory subsystem for future multicore systems. Regarding $\mathrm{SpMxV}$, we expect that the kernel will scale better in Nehalem, especially if it is assured that data are distributed among NUMA nodes so that each thread accesses local memory.

\subsection{Matrix Suite}

Iterative use of $\mathrm{SpMxV}$ induces temporal locality to the application. Hence, the streaming behavior of the kernel is maintained only if the working set, and more specifically the matrix data, is significantly larger than the system's aggregate cache. For this reason, we build our matrix suite using matrices with a CSR working set larger than $4 \cdot 6=24 \mathrm{MiB}$, which is the greater aggregate cache for the systems used in our experimental evaluation. The resulting matrix set consists of 50 matrices which are listed in Table II. The table also includes their characteristics, and the achieved size reduction from the proposed methods. Size reduction for the CSR-DUVI method is not included, but can be derived from the sum of the corresponding size reductions for CSR-DU and CSR-VI.

The majority of the matrices represent real-world problems and were selected from the University of Florida Sparse Matrix Collection [Davis 1997]. Our suite includes the fdif $202 \times 202 \times 102$ matrix, which is a matrix obtained by a 5-pt finite difference problem for a $202 \times 202 \times 102$ regular grid created by SPARSKIT [Saad 1994], and two artificial matrices that represent the two 
Table II.

Matrix suite used for the experimental evaluation. The first columns contain information about each matrix: dim contains the number of rows and columns of the matrix in thousands (nrows $=n c o l s$, since we consider only square matrices), $n n z$ contains the number of nonzero elements in millions, size contains the matrix size in MiB when stored in CSR format and ttu contains the total-to-unique ratio for the values of each matrix. The last five columns show the size reduction achieved by various methods compared to CSR. For BCSR we selected the block shape that achieved maximum size reduction.

\begin{tabular}{|c|c|c|c|c|c|c|c|c|c|}
\hline \multicolumn{5}{|c|}{ matrix characteristics } & \multicolumn{5}{|c|}{ size reduction $(\%)$} \\
\hline \multirow{2}{*}{ name } & \multirow{2}{*}{$\begin{array}{l}\operatorname{dim} \\
/ 10^{3}\end{array}$} & \multirow{2}{*}{$\begin{array}{l}\mathrm{nnz} \\
/ 10^{6}\end{array}$} & \multirow{2}{*}{$\begin{array}{l}\text { size } \\
/ 1 \mathrm{MiB}\end{array}$} & \multirow{2}{*}{ ttu } & \multirow{2}{*}{$\begin{array}{l}\text { BCSR } \\
(\max )\end{array}$} & \multicolumn{3}{|c|}{ DU } & \multirow[t]{2}{*}{ VI } \\
\hline & & & & & & noseq & seq $=8$ & seq $=4$ & \\
\hline boneS10 & 914.9 & 55.5 & 638.3 & $1,386,710.6$ & 22.5 & 16.6 & 27.1 & 30.0 & 58.0 \\
\hline ldoor & 952.2 & 46.5 & 536.0 & 2.1 & 9.5 & 6.9 & 19.9 & 30.1 & - \\
\hline inline_1 & 503.7 & 36.8 & 423.3 & 2.0 & 22.4 & 4.7 & 15.8 & 22.8 & - \\
\hline fdif202x202x102 & $4,000.0$ & 27.8 & 333.9 & $6,960,000.0$ & -38.5 & 15.9 & 15.9 & 15.9 & 55.7 \\
\hline F1 & 343.8 & 26.8 & 308.4 & 2.1 & 22.3 & 5.8 & 16.2 & 21.0 & - \\
\hline rajat31 & $4,690.0$ & 20.3 & 250.4 & $5,098.2$ & -39.3 & 21.5 & 21.5 & 21.5 & 46.4 \\
\hline msdoor & 415.9 & 20.2 & 233.2 & 2.1 & 10.2 & 11.9 & 22.3 & 29.1 & - \\
\hline Freescale1 & $3,428.8$ & 18.9 & 229.6 & 2.0 & -31.7 & 0.5 & 0.5 & 0.6 & - \\
\hline Ga41As41H72 & 268.1 & 18.5 & 212.6 & 5.1 & -16.9 & 16.6 & 21.7 & 25.1 & 20.3 \\
\hline af_shell9 & 504.9 & 17.6 & 203.2 & 18.2 & 12.0 & 16.6 & 28.8 & 30.9 & 29.4 \\
\hline af_5_k101 & 503.6 & 17.6 & 202.8 & 1.9 & 12.1 & 16.5 & 28.8 & 30.9 & - \\
\hline TSOPF_RS_b2383 & 38.1 & 16.2 & 185.2 & 21.2 & 26.3 & 21.0 & 33.1 & 33.1 & 30.2 \\
\hline kkt_power & $2,063.5$ & 14.6 & 175.1 & 173.5 & -61.2 & 5.2 & 5.2 & 5.2 & 31.5 \\
\hline Si41Ge41H72 & 185.6 & 15.0 & 172.5 & 3.2 & -13.5 & 16.6 & 21.8 & 25.7 & - \\
\hline random 100000 & 100.0 & 15.0 & 171.8 & $1,497.8$ & -66.3 & 16.7 & 16.7 & 16.7 & - \\
\hline nd12k & 36.0 & 14.2 & 162.9 & 2.9 & 16.4 & 16.7 & 29.3 & 30.0 & - \\
\hline crankseg_2 & 63.8 & 14.1 & 162.2 & 3.2 & 5.8 & 16.8 & 25.8 & 28.7 & - \\
\hline pwtk & 217.9 & 11.6 & 134.0 & 2.1 & 14.1 & 15.7 & 31.3 & 31.6 & - \\
\hline bmw3_2 & 227.4 & 11.3 & 130.1 & 2.7 & 7.4 & 17.5 & 25.9 & 30.0 & - \\
\hline ohne2 & 181.3 & 11.1 & 127.3 & 2.1 & -24.8 & 16.7 & 17.8 & 20.0 & - \\
\hline hood & 220.5 & 10.8 & 124.1 & 2.1 & 10.4 & 11.3 & 22.5 & 29.3 & - \\
\hline Si87H76 & 240.4 & 10.7 & 122.9 & 31.9 & -29.6 & 16.6 & 20.4 & 22.7 & 31.0 \\
\hline bmwcra_1 & 148.8 & 10.6 & 122.4 & 3.4 & 22.4 & 16.7 & 25.7 & 28.4 & - \\
\hline atmosmodj & $1,270.4$ & 8.8 & 105.7 & $2,203,720.0$ & -38.4 & 16.0 & 16.0 & 16.0 & 55.7 \\
\hline thermal2 & $1,228.0$ & 8.6 & 102.9 & 1.8 & -42.5 & 12.5 & 12.5 & 13.3 & - \\
\hline G3_circuit & $1,585.5$ & 7.7 & 93.7 & $31,787.7$ & -27.1 & 9.3 & 9.3 & 9.3 & 54.6 \\
\hline cage13 & 445.3 & 7.5 & 87.3 & $17,936.1$ & -56.2 & 11.1 & 11.1 & 11.1 & 49.0 \\
\hline rajat30 & 644.0 & 6.2 & 73.1 & 9.0 & -31.6 & 9.9 & 10.7 & 14.0 & 25.1 \\
\hline pre2 & 659.0 & 6.0 & 70.7 & 7.6 & -38.5 & 14.1 & 14.6 & 14.8 & 23.7 \\
\hline Hamrle3 & $1,447.4$ & 5.5 & 68.6 & $104,042.3$ & -6.2 & 17.8 & 17.8 & 19.6 & 53.6 \\
\hline largebasis & 440.0 & 5.6 & 65.3 & $17,539.7$ & 15.7 & 0.7 & 19.2 & 21.8 & 48.7 \\
\hline Chebyshev4 & 68.1 & 5.4 & 61.8 & 3.5 & -28.3 & 20.8 & 26.0 & 26.0 & - \\
\hline apache2 & 715.2 & 4.8 & 57.9 & $120,446.8$ & -37.7 & 15.9 & 15.9 & 15.9 & 55.6 \\
\hline s3dkq4m2 & 90.4 & 4.8 & 55.5 & 64.9 & 16.7 & 16.6 & 31.8 & 31.8 & 32.1 \\
\hline ship_001 & 34.9 & 4.6 & 53.3 & 3.8 & 6.2 & 16.8 & 28.7 & 31.2 & - \\
\hline torso3 & 259.2 & 4.4 & 51.7 & 1.4 & -25.4 & 15.3 & 15.3 & 15.3 & - \\
\hline thread & 29.7 & 4.5 & 51.3 & 2.1 & 22.3 & 17.1 & 26.5 & 28.2 & - \\
\hline ASIC_680k & 682.9 & 3.9 & 46.9 & 48.3 & -32.6 & 7.5 & 9.1 & 10.9 & 30.2 \\
\hline large-dense & 2.0 & 4.0 & 45.8 & 122.1 & 29.2 & 24.9 & 33.2 & 33.2 & - \\
\hline barrier2-9 & 115.6 & 3.9 & 45.0 & 3.6 & -37.6 & 16.8 & 16.8 & 17.3 & - \\
\hline
\end{tabular}

ACM Transactions on Architecture and Code Optimization, Vol. 7, No. 3, Article 16, Pub. date: December 2010. 
Table II. (Continued)

\begin{tabular}{|c|c|c|c|c|c|c|c|c|c|}
\hline \multicolumn{5}{|c|}{ matrix characteristics } & \multicolumn{5}{|c|}{ size reduction (\%) } \\
\hline \multirow{2}{*}{ name } & \multirow{2}{*}{$\begin{array}{l}\operatorname{dim} \\
/ 10^{3}\end{array}$} & \multirow{2}{*}{$\begin{array}{l}\mathrm{nnz} \\
/ 10^{6}\end{array}$} & \multirow{2}{*}{$\begin{array}{l}\text { size } \\
/ 1 \mathrm{MiB}\end{array}$} & \multirow{2}{*}{ ttu } & \multirow{2}{*}{$\begin{array}{l}\text { BCSR } \\
(\max )\end{array}$} & \multicolumn{3}{|c|}{ DU } & \multirow[t]{2}{*}{ VI } \\
\hline & & & & & & noseq & seq $=8$ & seq $=4$ & \\
\hline xenon2 & 157.5 & 3.9 & 44.9 & 41.4 & 19.4 & 21.0 & 21.0 & 21.8 & 31.3 \\
\hline parabolic_fem & 525.8 & 3.7 & 44.1 & 14.2 & -46.9 & 1.0 & 1.0 & 1.0 & 27.3 \\
\hline FEM_3D_thermal2 & 147.9 & 3.5 & 40.5 & 1.9 & -13.7 & 19.3 & 19.3 & 19.3 & - \\
\hline sme3Dc & 42.9 & 3.1 & 36.2 & 1.3 & -58.0 & 16.8 & 16.8 & 16.8 & - \\
\hline stomach & 213.4 & 3.0 & 35.4 & 1.3 & -29.7 & 21.5 & 21.5 & 21.5 & - \\
\hline thermomech_dK & 204.3 & 2.8 & 33.4 & 1.4 & 25.6 & 12.8 & 12.8 & 13.1 & - \\
\hline helm2d03 & 392.3 & 2.7 & 32.9 & 25.0 & -52.1 & 1.4 & 1.4 & 3.8 & 29.3 \\
\hline ASIC_680ks & 682.7 & 2.3 & 29.3 & 57.2 & -31.7 & 17.8 & 20.3 & 22.3 & 44.5 \\
\hline poisson3Db & 85.6 & 2.4 & 27.5 & 1.0 & -60.0 & 17.1 & 17.1 & 17.1 & - \\
\hline rma10 & 46.8 & 2.4 & 27.3 & 1.9 & 5.3 & 19.1 & 26.1 & 29.4 & - \\
\hline
\end{tabular}

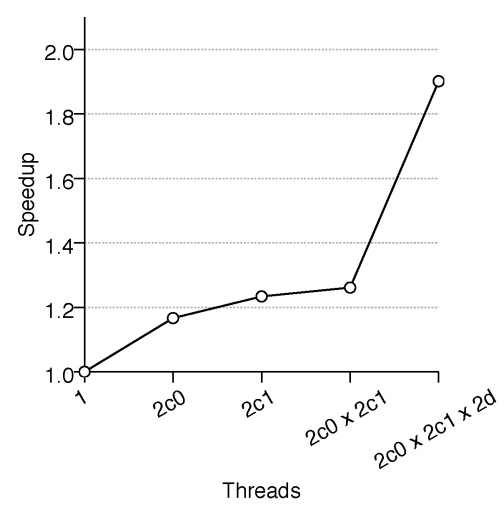

(a) Harpertown

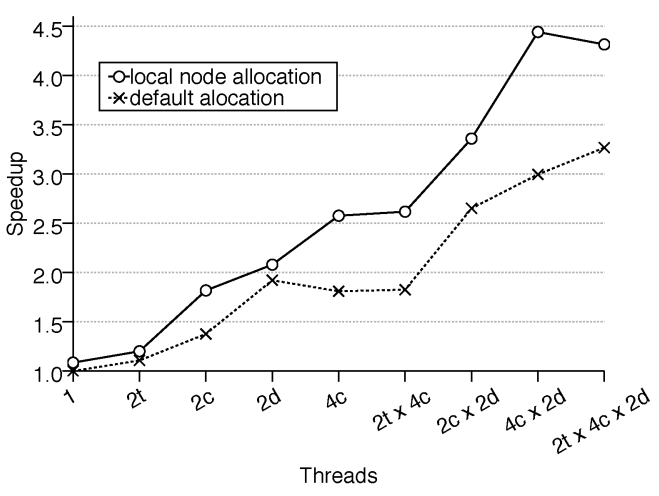

(b) Nehalem

Fig. 14. Average speedups for the multithreaded CSR SpMxV kernel across different thread configurations. The "local node allocation" line in the Nehalem figure corresponds to a NUMAaware version of the kernel, that binds matrix data in local NUMA node memory. The speedup for Nehalem is obtained using the single-threaded performance with default allocation, as the base performance.

ends of the sparsity spectrum: (a) a dense $2000 \times 2000$ matrix (large-dense) and $(b)$ a random $100000 \times 100000$ sparse matrix (random100000).

\subsection{CSR Performance Evaluation}

Figure 14(a) illustrates the average speedup of multithreaded CSR over all matrices, for different thread scheduling configurations on the Harpertown system. The speedup for 8 threads is 1.9 , demonstrating the poor scalability of $\mathrm{SpMxV}$. The speedup increase observed between the 2c0 and $2 \mathrm{c} 1$ cases, 1.17 and 1.23 respectively, can be accredited to matrix data caching during consecutive $\mathrm{SpMxV}$ executions. Cases $2 \mathrm{c} 1$ and $2 \mathrm{c} 0 \times 2 \mathrm{c} 1$ achieve roughly the same performance, even though available processors are doubled. We attribute this fact to the limited memory bandwidth since, as is shown in Figure 12, the 
available memory throughput for 2 and 4 cores in a single die is essentially the same.

A NUMA-oblivious multithreaded program can run unmodified in a NUMA system. However, there is no guarantee that data placement will be efficient. Thus, to maximize performance, we developed NUMA-aware versions of our methods, where memory allocation ensures that data accessed from a single thread are placed into the local NUMA node of the corresponding processor. Data shared between threads, e.g., the unique values array in the CSR-VI and CSR-DUVI methods, are allocated using standard mechanisms (i.e., malloc()).

Figure 14(b) presents results for the Nehalem system for two versions of the CSR SpMxV kernel: default allocation (NUMA-oblivious) and local allocation (NUMA-aware). The large memory throughput capabilities of Nehalem result in noticeably better performance than Harpertown, even for the NUMAoblivious version. The NUMA-aware version further improves performance, achieving a 4.44 speedup for the $4 \mathrm{c} \times 2 \mathrm{~d}$ case. SMT threads utilization in this case, however, degrades performance (4.31).

Even though the Nehalem memory subsystem architecture drastically increases CSR SpMxV performance, it is still far from the theoretical maximum, leaving room for performance improvement by applying compression schemes. In the following sections we present only NUMA-aware versions for all methods on the Nehalem system to focus on cases that maximize performance.

\subsection{CSR-DU Performance Evaluation}

To evaluate CSR-DU, we performed experimental runs using several different combinations for the method's parameters. Versions with aligned deltas and unit offsets performed consistently better or similar than the rest, and so we present only them in the following results. Regarding sequential units, we consider three cases: absence of sequential units (noseq) and sequential units with a minimum of $8(s e q=8)$ and $4(s e q=4)$ elements. It should be noted that $s e q=4$ performs more aggressive compression than $s e q=8$. We compare CSR-DU performance against both CSR and BCSR. For the BCSR method we performed experiments with a number of different block shapes configurations, ${ }^{4}$ using specialized $\mathrm{SpMxV}$ versions. In the following results, unless otherwise stated, we use the best performing BCSR case over all available block shape configurations.

The compression ratios achieved for each matrix are listed in Table II. The size reduction achieved for the large-dense matrix is the maximum possible for CSR-DU: $24.9 \%$ for noseq, and $33.2 \%$ for sequential units. The compression ratios of other matrices in our suite show a large variation, ranging from zero to close to maximum. On average, CSR-DU reduces matrix data by $14.2 \%$ for noseq, $19.3 \%$ for $s e q=8$ and $21.1 \%$ for $s e q=4$. BCSR is not able to efficiently capture the structure of matrices in our suite: it results in size increase for 28 matrices. Moreover, only for 2 matrices (F1, thermomech_dK) the best BCSR reduction is greater than reduction achieved by CSR-DU seq $=4$.

${ }^{4}$ The block shapes considered were: $1 \times 2,1 \times 3,1 \times 4,2 \times 1,2 \times 2,2 \times 3,2 \times 4,3 \times 1,3 \times 2,4 \times 1$, $4 \times 2$.

ACM Transactions on Architecture and Code Optimization, Vol. 7, No. 3, Article 16, Pub. date: December 2010. 


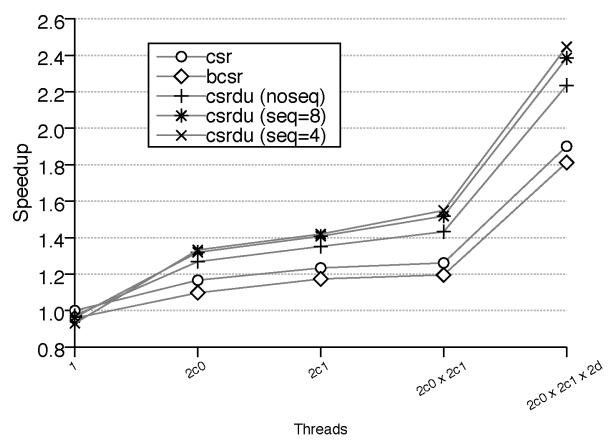

(a)

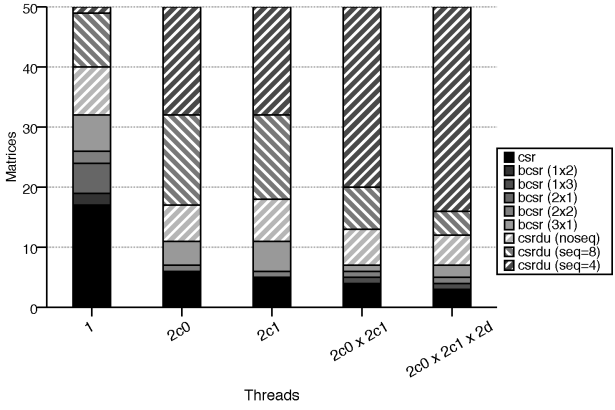

(b)
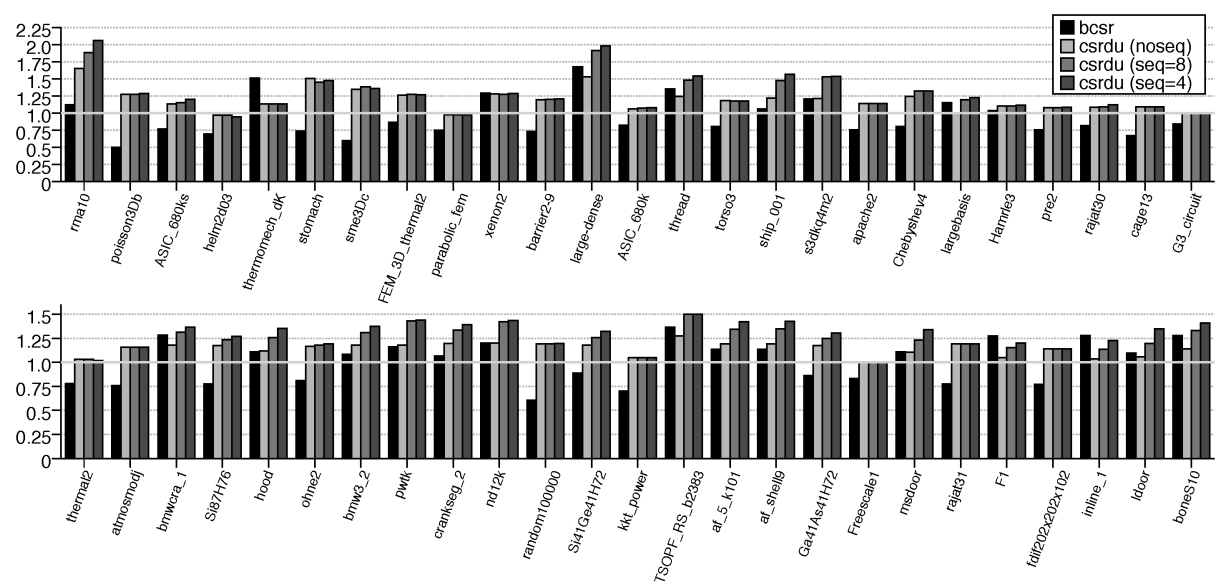

(c)

Fig. 15. CSR-DU performance results for the Harpertown system: (a) Average speedup of CSR, BSCR and CSR-DU methods over serial CSR for different thread configurations. (b) Distribution of best performing methods for different thread configurations. (c) performance improvement of BCSR and CSR-DU methods over CSR for individual matrices when all 8 cores are utilized. For (a) and (c), the BCSR performance is the best performing case for each matrix over all considered block shapes.

Figure 15 illustrates Harpertown CSR-DU performance results. Figure 15(a) shows the average speedup of CSR, BCSR, and CSR-DU over single-threaded CSR, for different thread affinity configurations. BCSR performs worse than CSR on average for all cases. The reason for that is the large number of matrices for which BCSR results in size increase. When all cores are utilized, CSR-DU methods perform better on average than CSR and BCSR. The $s e q=4$ case achieves the best average speedup for 8 threads (2.45), improving performance by $28.7 \%$ and $35.0 \%$ over CSR and BCSR average, respectively. An interesting aspect of CSR-DU variants performance is that the best version for 8 threads $(s e q=4)$ has the lowest performance in the serial case (7\% slowdown compared to CSR). 
Figure 15(c) shows the performance improvement of BCSR and CSR-DU over CSR for each individual matrix, when 8 threads are utilized. An important observation is that the CSR-DU method does not exhibit significantly reduced performance over CSR for any matrix in our suite. For BCSR, a significant number of matrices take a performance hit compared to CSR, due to a significant size increase.

Selecting the optimal storage format is not a straightforward task, since performance depends on the system architecture and the matrix structure in ways that are not always visible or simple. Figure 15(b) depicts a breakdown of the best performing methods distribution for our matrix suite. Concentrating on the full core utilization case for Harpertown, CSR-DU is a good universal choice for our matrix suite, since it achieves best performance for 43 matrices. For the same case, BCSR achieves the best performance for 4 matrices and CSR for 3. When only one thread is utilized, the best performing methods distribution is more balanced (17 for CSR, 15 for BCSR and 18 for CSR-DU). In general, we argue that as long as the main bottleneck is memory bandwidth, CSR-DU is a more promising option than BCSR and CSR. When the memory bandwidth bottleneck becomes less severe, the computation overhead imposed by decompression is not amortized, making CSR-DU less attractive.

Nehalem results are presented in Figure 16. Figure 16(a) demonstrates the average speedup of considered methods over serial CSR. When all cores are utilized $(4 c \times 2 d)$, the best average performance for CSR-DU is 4.6 , a $12.2 \%$ and $11.7 \%$ improvement over CSR and BCSR averages respectively, and is achieved by seq $=8$. Hence, even for the Nehalem system, where the memory bottleneck is less severe, there is a margin for performance improvement by applying CSR-DU. Interestingly, when all SMT threads are utilized $(2 \mathrm{t} \times 4 \mathrm{c} \times 2 \mathrm{~d})$, CSR-DU average for seq $=4$ and seq $=8$ is improved; all other methods result in a slowdown.

Figure 16(c) presents BCSR and CSR-DU improvement over CSR for each matrix in our suite when all threads are utilized $(2 t \times 4 c \times 2 d)$. CSR-DU performs worse than CSR for only five matrices (helm2d03, sme3Dc, parabolic_fem, G3_circuit, Freescale1) and in some cases the difference is marginal. Thus, we argue that, CSR-DU is fairly stable, even when the memory bottleneck is alleviated by the system's architectural characteristics. The number of matrices that exhibit reduced performance for BCSR is 24 , which again can be attributed to padding.

Figure 15(b) illustrates the distribution of best performing methods. For a single thread, CSR achieves the best performance for 19 matrices, BCSR for 22 and CSR-DU for 9. However, as the number of cores utilized increases, CSR-DU becomes the most attractive option: for $4 \mathrm{c} \times 2 \mathrm{~d}$ CSR-DU performs best for 36 matrices, BCSR for 9 and CSR for 5 .

\subsection{CSR-VI Performance Evaluation}

Contrary to CSR-DU, CSR-VI can be applied meaningfully only to matrices with a large number of common values. To elaborate on the applicability of the method for a given matrix, we define the total-to-unique (ttu) values ratio, 


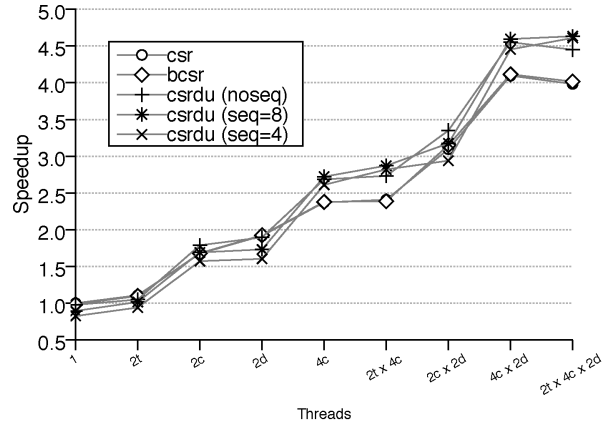

(a)

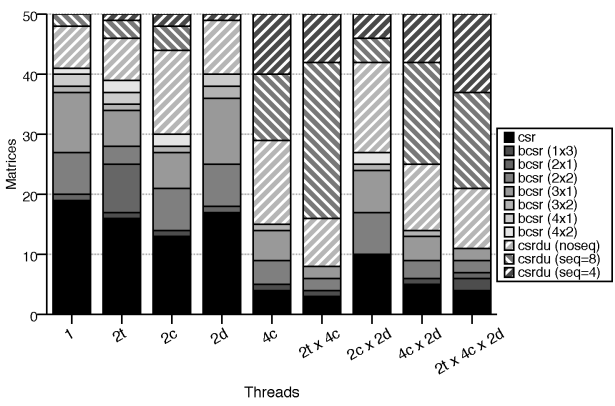

(b)

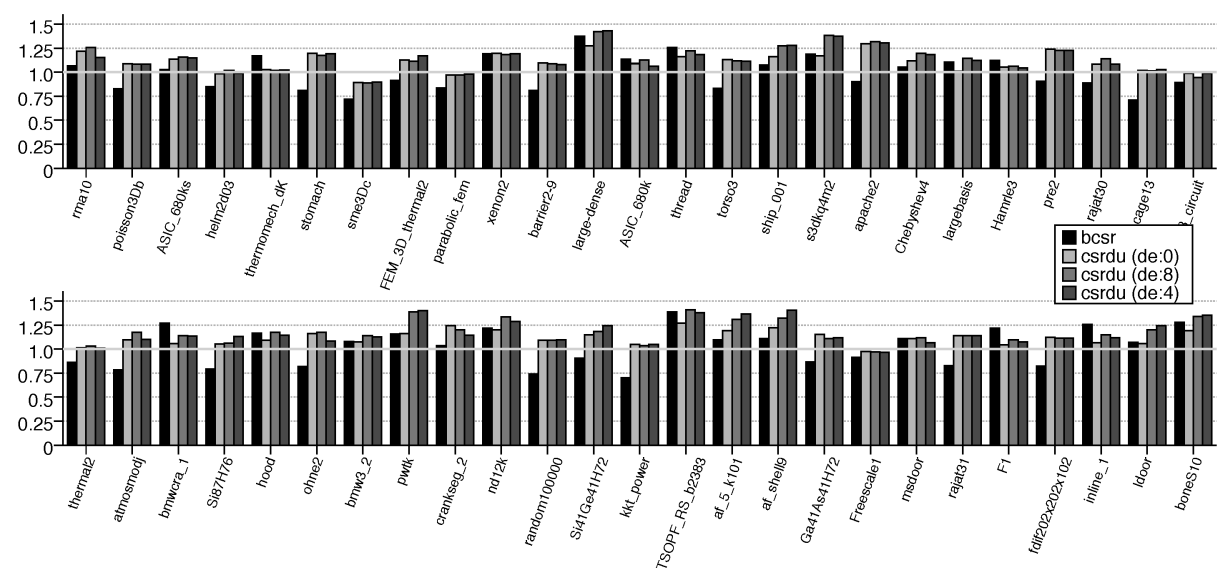

(c)

Fig. 16. CSR-DU performance results for the Nehalem system: (a) Average speedup of CSR, BCSR and CSR-DU methods over serial CSR for different thread configurations. (b) Distribution of best performing methods for different thread configurations. (c) performance improvement of BCSR and CSR-DU methods over CSR for individual matrices, when all 16 threads are utilized $(2 t \times 4 c \times 4 d)$. All methods considered are NUMA-aware. For (a) and (c), the BCSR performance is the best performing case for each matrix over all considered block shapes.

as the fraction of the number of nonzero elements $(n n z)$ to the number of unique values (uvals), that is, $t t u=\frac{n n z}{\text { uvals }}$. A high value of $t t u$ indicates that the matrix is fitting for the CSR-VI method, while a small one shows that it will most likely result in slowdown. We use the empirical criterion $t t u \geq 5$ to select the appropriate matrices from our matrix suite, and discard artificial matrices random 100000 and large-dense which have randomly created values. The resulting matrix suite has 22 matrices, which is a significant portion of the original set (see Table II). For these matrices, CSR-VI achieves an average matrix size reduction of $39.2 \%$, the maximum and the minimum being $58.0 \%$ (boneS10) and 20.3\% (Ga41As41H72), respectively.

Performance results for Harpertown are shown in Figure 17. As expected, performance gains from value compression are larger than those from index 


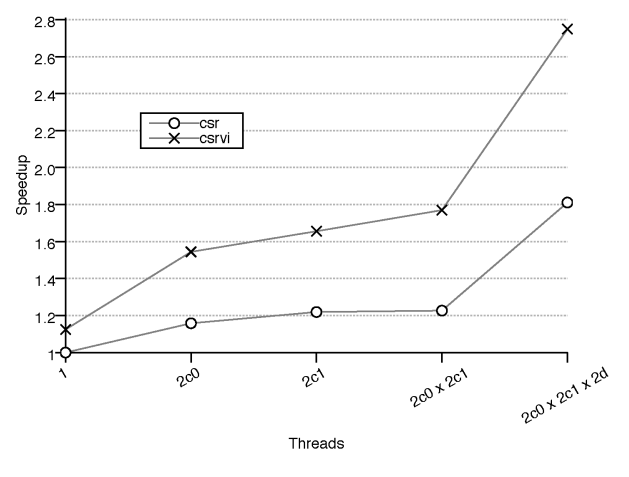

(a)

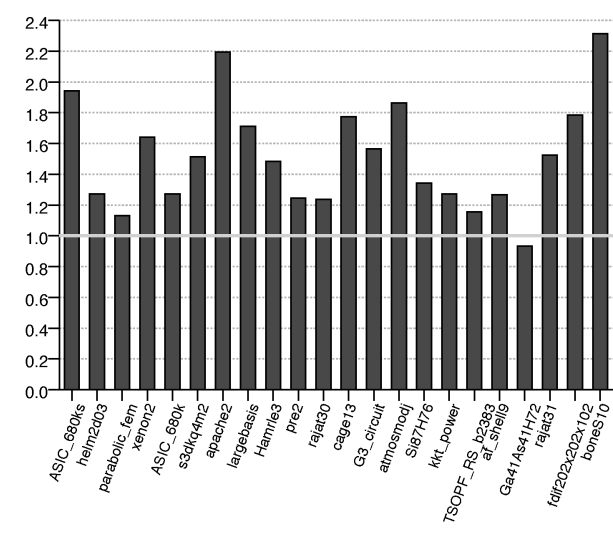

(b)

Fig. 17. Performance of the CSR-VI method on the Harpertown system: (a) average speedup of CSR and CSR-VI methods for different thread configurations. (b) performance improvement of the CSR-VI method over CSR for individual matrices, when all 8 cores are utilized.

compression, since we are considering 32-bit indices and 64-bit values for our reference CSR implementation. Even in the serial case, CSR-VI achieves a 12.4\% performance improvement over CSR (Figure 17(a)). As the number of utilized cores increases, memory bandwidth bottleneck becomes more intense and working set reduction becomes more beneficial. For 8 threads the average CSR-VI speedup is 2.75 , which is a $51.7 \%$ improvement over the corresponding CSR case. Figure 17(b) shows the performance improvement of CSR-VI over CSR for each individual matrix, when using 8 threads. CSR-VI leads to reduced performance for only Ga41As41H72, which is the matrix with the lower $t t u$ value (5.1) in our suite.

CSR-VI performance for Nehalem is shown in Figure 18. The ample memory throughput capabilities of Nehalem limit the potential CSR-VI benefits. CSR is able to utilize a large portion of these capabilities due to hardware prefetching. This technique, employed by modern processors, detects easily predicted memory access patterns (e.g., sequential) and prefetches successive data into the cache hierarchy. Contrarily, CSR-VI performs random accesses on the vals_unique array; these accesses cannot be predicted, leading to increased memory latencies.

As can be seen in Figure 18(a), CSR-VI performs worse than CSR in the serial case (slowdown of $15 \%$ ). In the $4 \mathrm{c} \times 2 \mathrm{~d}$ case, however, CSR-VI reaches a speedup of 4.13 , which is a $8.6 \%$ improvement over the corresponding CSR average speedup. When all available SMT threads at each core are utilized $(2 t \times 4 c \times 2 d)$ CSR-VI average is increased to 4.23 . For $2 \mathrm{t} \times 4 \mathrm{c} \times 2 \mathrm{~d}$, CSR-VI performs worse than CSR for 6 matrices (Figure 18(b)).

\subsection{CSR-DUVI Performance Evaluation}

Combining CSR-VI and CSR-DU leads to an average size reduction of 52.4\% for noseq, $56.2 \%$ for $s e q=8$ and $57.3 \%$ for $s e q=4$. Experimental results for 


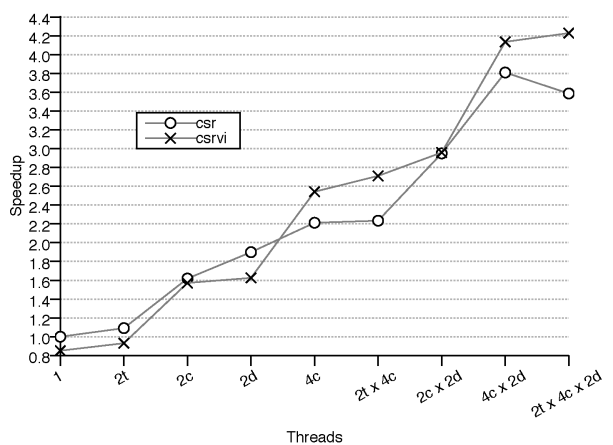

Threads

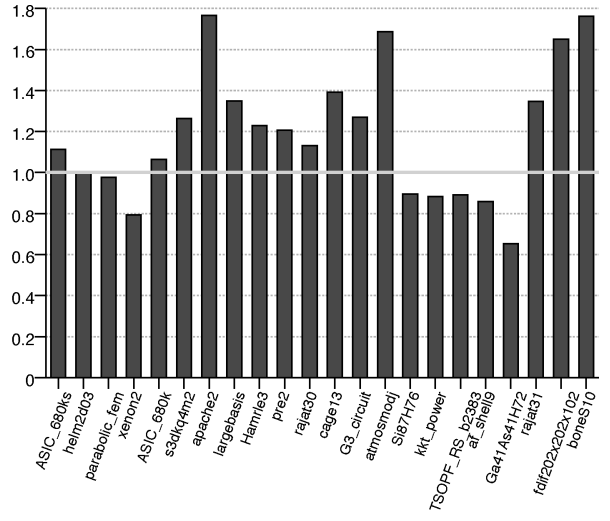

(b)

Fig. 18. Performance of the CSR-VI method on the Nehalem system: (a) average speedup of CSR and CSR-VI methods for different thread configurations. (b) performance improvement of the CSR-VI method over CSR for individual matrices, when all 16 threads are utilized. All methods considered are NUMA-aware.

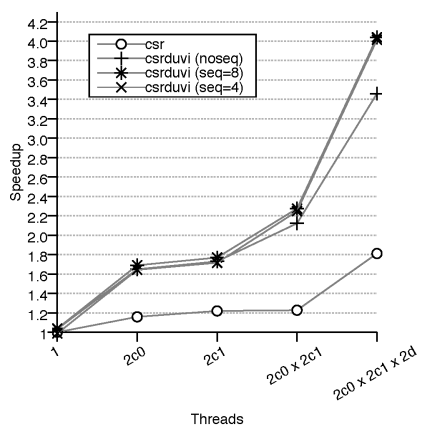

(a)

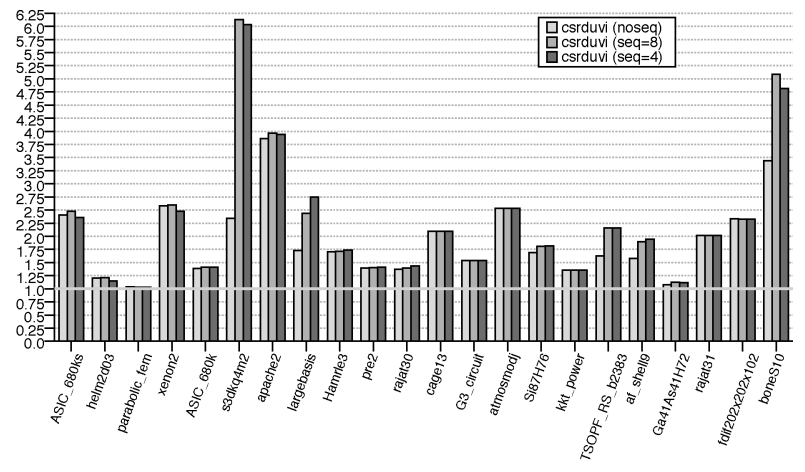

(b)

Fig. 19. Performance of the CSR-DUVI method on the Harpertown system: (a) speedup of CSR and CSR-DUVI methods over serial CSR for different thread configurations. (b) performance improvement of CSR-DUVI methods over CSR for individual matrices, when all 8 cores are utilized.

Harpertown are illustrated in Figure 19. As can be seen in Figure 19(a), CSR-DUVI serial performance is similar to CSR. In the $2 \mathrm{c} 0 \times 2 \mathrm{c} 1 \times 2 \mathrm{~d}$ case, however, CSR-DUVI results in a significant parallel speedup increase. More specifically, $s e q=8$ achieves a speedup of 4.04 , which improves upon CSR and CSR-VI by $123 \%$ and $47 \%$, respectively. Evidently, part of this large improvement is due to matrices which now fit, in whole or in a significant portion, into L2 cache. Moreover, as can be seen in Figure 19(b), CSR-DUVI improves performance over CSR for all matrices.

Nehalem results are presented in Figure 20. The best CSR-DUVI average speedup for $4 \mathrm{c} \times 2 \mathrm{~d}(4.41)$ and $2 \mathrm{t} \times 4 \mathrm{c} \times 2 \mathrm{~d}$ (4.57) is achieved by $s e q=8$ (Figure 20(a)). The respective improvements over CSR-VI are $6.6 \%$ and $8.2 \%$, and 


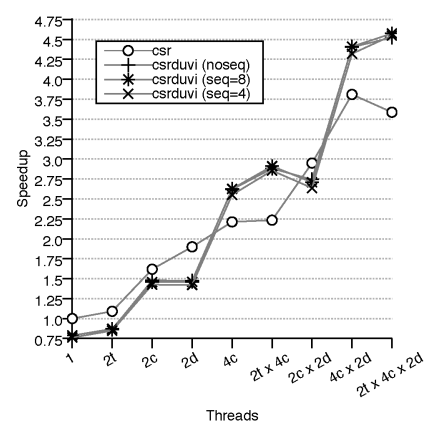

(a)

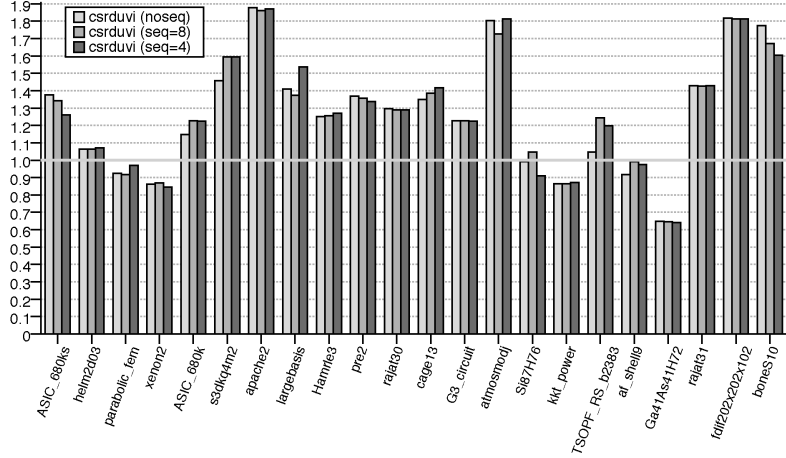

(b)

Fig. 20. Performance of the CSR-DUVI method on the Nehalem system: (a) speedup of CSR and CSR-DUVI methods over serial CSR for different thread configurations (b) performance improvement of CSR-DUVImethods over CSR for individual matrices, when all 16 threads are utilized. All methods are NUMA-aware.

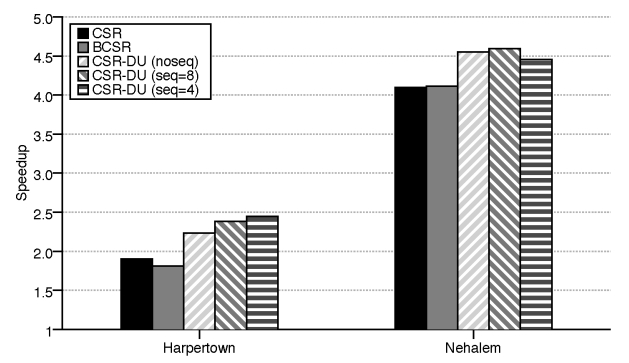

(a)

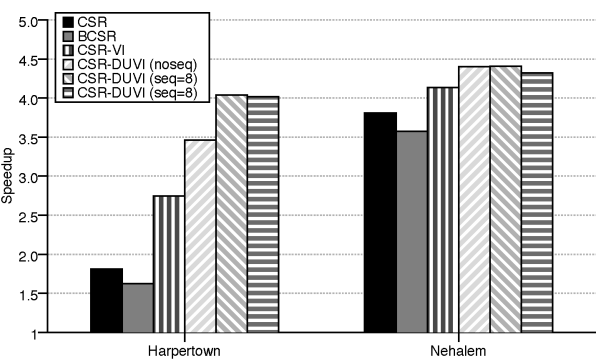

(b)

Fig. 21. Average speedups over the serial CSR for various methods, when all cores are utilized $(2 c 0 \times 2 c 1 \times 2 d$ for Harpertown and $4 c \times 2 d$ for Nehalem): (a): CSR, BCSR and CSR-DU over all matrices, (b): CSR, BCSR, CSR-VI, CSR-DUVI over the 22 matrices with $t t u \geq 5$. The Nehalem versions are NUMA-aware, to take full advantage of the underlying hardware.

over CSR's best performing case $(4 \mathrm{c} \times 2 \mathrm{~d}) 15.7 \%$ and $20 \%$. Finally, there are 5 matrices with reduced CSR-DUVI performance over CSR (Figure 19(b)).

\subsection{Performance Evaluation Summary}

Figure 21 summarizes previous results. It illustrates the average speedup over single-threaded CSR for all methods examined in both systems, when all 8 cores are utilized. A general conclusion is that there exists a compression level, different for each architecture, beyond which the tradeoff between storage and computation becomes less attractive and can even lead to performance degradation. For the Harpertown processor, which has limited available bandwidth, this point is difficult to reach and it only becomes visible in our experiments in the CSR-DUVI method where the performance of $s e q=8$ is better than the $s e q=4$ case. On the other hand, the architectural characteristics of the Nehalem system (NUMA, larger memory throughput capabilities) 
make this point easier to reach (e.g., small improvement of CSR-DUVI over CSR-VI).

A question that rises, regarding the applicability of compression methods for improving $\mathrm{SpMxV}$ performance, is whether they will remain profitable on the face of new systems that focus on memory throughput performance. An argument in favor of such methods is that as memory throughput increases, so does the number of the cores on the system (e.g., the Nehalem architecture targets 8-core processors, which have not yet been introduced in the market). Moreover, memory performance tends to improve with smaller rates than CPU performance (memory wall problem).

\section{RELATED WORK}

\subsection{Serial SpMxV}

Due to the importance of $\mathrm{SpMxV}$ there is an abundance of scientific work targeting the optimization of the serial version of the kernel. A number of alternatives to CSR have been proposed, such as JD (Jagged Diagonal), CDS (Compressed Diagonal Storage) and Ellpack-Itpack [Barrett et al. 1994; Saad 2003]. These formats try to exploit regularities in the structure of the sparse matrix, to reduce the storage requirements and the execution time of $\mathrm{SpMxV}$. Moreover, there is a large number of works that propose optimization techniques for the efficient execution of the kernel. Several of these efforts [Toledo 1997; Pinar and Heath 1999; Im and Yelick 2001; Vuduc et al. 2002; Vuduc and Moon 2005] aim at the optimization of the irregular and indirect accesses on the $x$ vector, using methods such as matrix reordering, register blocking and cache blocking. Other works [White and Sadayappan 1997; Mellor-Crummey and Garvin 2004] are concerned with the performance problems that arise in matrices with a large number of rows with small length.

\subsection{Index Compression}

A significant part of the $\mathrm{SpMxV}$ optimization techniques reported in the related literature result in index data reduction. Typical examples are blocking methods such as BCSR [Saad 1994; Im and Yelick 2001] that store only per-block index information. Traditionally the main focus of BCSR has been serial performance improvement (e.g., via register blocking) and not working set reduction. For this reason, BCSR has several disadvantages if used as a compression technique. First and foremost, depending on the matrix structure, it may increase the total size of the matrix due to padding (Table II). Secondly, it relies on constant-shape blocks and thus has limited capabilities of adapting to more complex matrix structures. Pinar and Heath [1999] describe a one-dimensional variable block scheme, similar to the one used for CSR-DU sequential units. They also discuss column reordering techniques that aim to align the nonzero elements in a row in consecutive locations as much as possible. CSR-DU could benefit from similar reordering techniques towards two directions: (a) creating larger sequential units and (b) creating denser units that require smaller delta values. Although not equivalent, the latter is strongly related to 
matrix bandwidth reduction techniques than have been extensively studied in the past because they relate to $\mathrm{SpMxV}$ cache performance [Temam and Jalby 1992; Pichel et al. 2004].

One of the few works that explicitly targets the compression of the index data is Willcock and Lumsdaine [2006]. In that paper, Willcock and Lumsdaine propose two methods: DCSR, which compresses column indices using a byteoriented delta encoding scheme to exploit the highly redundant nature of the col_ind array and RPCSR, which generates matrix-specific dynamic code by applying aggressive compression on column indices patterns for the whole matrix. We will focus our comparison on the DCSR method, which operates on the same level as CSR-DU. DCSR encodes the matrix using a set of six command codes for primitive suboperations that can be used to implement the $\mathrm{SpMxV}$ kernel. Examples of such suboperations are the increment of the current row and column index, and the multiplication of a number of the matrix values with the appropriate vector elements. A significant performance problem of this approach is that the decoding of these suboperations must be performed very often, which results in frequent mispredicted branches. This problem is dealt by a form of unrolling where patterns of frequent instances of six of these suboperations are grouped together allowing them to be executed sequentially, that is, without branches. Contrarily, our approach, which is also based on delta encoding, tackles the problem of branch misprediction performance penalties in a more basic level by being more coarse-grained. This allows for a much simpler and general implementation, while sustaining a small performance gain gap compared to the DCSR method. Moreover, it can handle worst-case scenarios of the DCSR method such as matrices that exhibit large variation with regard to the patterns encountered. A more detailed comparison of the two methods can be found in Kourtis et al. [2008b]. Another recent work that targets performance improvement by reducing the index data volume is Belgin et al. [2009], which proposes a matrix representation that exploits repeated block patterns. The authors search for frequently met block patterns and generate specialized inner loops for those, on top of a dispatch logic. They provide an evaluation of a parallel version, but they focus primarily on serial performance.

\subsection{Value Compression}

Despite that, in the common case, the value data constitute the larger part of the working set of $\mathrm{SpMxV}$, there has been little research effort targeting its reduction. Lee et al. [2004] exploit matrix symmetry by storing only half the matrix, that is, reducing matrix data volume by $50 \%$. However, our methods can lead to larger than 50\% size reduction. For example, CSR-DUVI applied to the symmetric matrix boneS10 leads to a reduction of $88.1 \%$. In the context of specialized hardware accelerators for $\mathrm{SpMxV}$, Moloney et al. [2005] discuss compression techniques for both index and value data. Additionally, there exist a number of works in the general area of scientific computation that are related to the value compression for the $\mathrm{SpMxV}$ kernel. Keyes [2000], proposes the use of lower precision representation for data that do not pose problems in the convergence procedure, while Langou et al. [2006] propose mixed precision 
algorithms, which deliver double precision arithmetic, while performing the bulk of the work in single precision. Even though these approaches target more on the exploitation of characteristics of modern architectures (e.g., vectorization), they also contribute significantly to the required memory bandwidth reduction. In a different context Burtscher and Ratanaworabhan [2007] propose a method for the efficient compression of double precision floating point values targeting network data transfers.

\subsection{Multithreaded SpMxV}

As far as the multithreaded version of the code is concerned, past work focuses mainly on SMP clusters where researchers either apply and evaluate known uniprocessor optimization techniques (e.g., register and cache blocking) on SMPs, or examine reordering techniques to improve locality of references and minimize communication cost [Im and Yelick 1999; Geus and Röllin 1999; Pichel et al. 2004; Catalyuerek and Aykanat 1996]. Williams et al. [2007] present an evaluation of $\mathrm{SpMxV}$ on a set of emerging multicore architectures. Their study covers a wide and diverse range of high-end chip multiprocessors, including recent multicores from AMD (Opteron X2) and Intel (Clovertown), Sun's Niagara2 and platforms comprised of one or two Cell processors. Their work includes a rich collection of optimizations, including some that are targeted specifically at multithreading architectures on a set of 14 matrices. In their conclusions they state that memory bandwidth could be a significant bottleneck and advocate working set reduction techniques. It should also be noted that one of the optimizations they apply is a simple index reduction technique, in which 16-bit indices are used when this is applicable.

\section{CONCLUSIONS}

To improve the performance of the multithreaded $\mathrm{SpMxV}$ kernel, by alleviating the contention on the memory subsystem, we proposed two sparse matrix storage formats: CSR-DU and CSR-VI, that apply compression to the index and value data of the matrix respectively. More specifically, CSR-DU applies a coarse-grained delta encoding compression scheme for column indices, and optionally supports dense one-dimensional blocks of variable length. CSR-VI, on the other hand, uses indirect indexing for the numerical value data, and can be meaningfully applied to matrices that exhibit a large percentage of common values. Moreover, we also considered the combination of these two formats (CSR-DUVI), that employs both the aforementioned techniques.

We performed an experimental evaluation on two different multicore systems: a typical SMP system (Harpertown), and a NUMA system (Nehalem). We used a rich matrix set, with working sets large enough to preserve the memory bound nature of the kernel. All methods demonstrated a noticeable improvement over CSR and BCSR in both systems, when all available cores were employed. The improvements were, as expected, larger on the Harpertown system, which also favors more aggressive compression techniques, due to its restricted memory bandwidth. Additionally, our proposed methods 
exhibited performance stability, since only a small subset of our suite resulted in a significant slowdown compared to CSR.

In conclusion, as the number of processing cores increases, simultaneous access on the shared system's memory will be the primary performance restraining factor for applications with streaming memory access patterns, such as $\mathrm{SpMxV}$. Hence, we argue that our approach designates a general optimization methodology for memory intensive problems, where compression sacrifices CPU cycles to alleviate memory pressure. As we have demonstrated for the SpMxV kernel, compression can potentially lead to substantial performance improvements in multithreaded execution, even if it leads to slowdowns in the uniprocessor case. However, not all memory intensive applications are suitable for this technique. For compression to be beneficial in this context, data should be fetched from memory multiple times, so that compression overhead is amortized. Possible suitable kernels can be sought in application domains such as graph and database algorithms.

\section{ACKNOWLEDGMENTS}

The authors wish to express their gratitude to the anonymous reviewers, whose suggestions greatly improved the quality of this manuscript.

\section{REFERENCES}

Anderson, W. K., Gropp, W. D., Kaushik, D. K., Keyes, D. E., and Smith, B. F. 1999 . Achieving high sustained performance in an unstructured mesh cfd application. In Proceedings of the ACM/IEEE Conference on Supercomputing (SC'99). ACM, New York, NY, 69.

Asanovic, K., Bodik, R., Catanzaro, B. C., Gebis, J. J., Husbands, P., Keutzer, K., Patterson, D. A., Plishker, W. L., Shalf, J., Williams, S. W., and Yelick, K. A. 2006. The landscape of parallel computing research: a view from Berkeley. Tech. rep. UCB/EECS-2006-183, EECS Department, University of California, Berkeley.

Barker, K., Davis, K., Hoisie, A., Kerbyson, D. J., Lang, M., Pakin, S., and Sancho, J. C. 2008. A performance evaluation of the Nehalem quad-core processor for scientific computing. Parall. Process. Lett.

Barrett, R., Berry, M., Chan, T. F., Demmel, J., Donato, J. M., Dongarra, J., EiJkhout, V., Pozo, R., Romine, C., AND DER Vorst, H. V. 1994. Templates for the Solution of Linear Systems: Building Blocks for Iterative Methods. SIAM, Philadelphia, PA.

Belgin, M., Back, G., And Ribbens, C. J. 2009. Pattern-based sparse matrix representation for memory-efficient smvm kernels. In Proceedings of the 23rd International Conference on Supercomputing (ICS'09). ACM, New York, NY, 100-109.

Burtscher, M. and Ratanaworabhan, P. 2007. High throughput compression of double-precision floating-point data. In Proceedings of the Data Compression Conference (DCC'07). IEEE Computer Society, Los Alamitos, CA, 293-302.

Catalyuerek, U. V. and Aykanat, C. 1996. Decomposing irregularly sparse matrices for parallel matrix-vector multiplication. Lecture Notes in Computer Science, vol. 1117, 75-86.

Culler, D. And Singh, J. 1999. Parallel Computer Architecture. Morgan Kaufmann Publishers San Francisco, CA.

DAVIS, T. 1997. University of Florida sparse matrix collection. NA Digest 97, 23, 7.

GEER, D. 2005. Chip makers turn to multicore processors. IEEE Comput. 38, 5, 11-13.

Geus, R. AND Röllin, S. 1999. Towards a fast parallel sparse matrix-vector multiplication. In Proceedings of the International Conference on Fundamentals and Applications of Parallel Computing (ParCo). Imperial College Press, 308-315.

ACM Transactions on Architecture and Code Optimization, Vol. 7, No. 3, Article 16, Pub. date: December 2010. 
Goumas, G., Kourtis, K., Anastopoulos, N., Karakasis, V., and Koziris, N. $2008 . \quad$ Performance evaluation of the sparse matrix-vector multiplication on modern architectures. $J$. Supercomput.

Gschwind, M., Hofstee, H. P., Flachs, B. K., Hopkins, M., Watanabe, Y., and Yamazaki, T. 2006. Synergistic processing in cell's multicore architecture. IEEE Micro 26, 2, 10-24.

Hennessy, J. and Patterson, D. 2007. Computer Architecture: A Quantitative Approach. Morgan Kaufmann.

Hsu, L., Iyer, R., Makineni, S., Reinhardt, S., and Newell, D. $2005 . \quad$ Exploring the cache design space for large scale CMPs. ACM SIGARCH Comput. Architect. News 33, 4, 24-33.

Im, E. AND Yelick, K. 1999. Optimizing sparse matrix-vector multiplication on SMPs. In Proceedings of the 9th SIAM Conference on Parallel Processing for Scientific Computing.

IM, E. AND YELICK, K. 2001. Optimizing sparse matrix computations for register reuse in SPARSITY. Lecture Notes in Computer Science, vol. 2073, 127-136.

KEYES, D. 2000. Four horizons for enhancing the performance of parallel simulations based on partial differential equations. In Proceedings of the 6th International Conference on Parallel Processing (Euro-Par'00).

Kourtis, K., Goumas, G., AND Koziris, N. 2008a. Improving the performance of multithreaded sparse matrix-vector multiplication using index and value compression. In Proceedings of the 37th International Conference on Parallel Processing (ICPP'08), 511-519.

KourTis, K., Goumas, G., AND Koziris, N. 2008b. Optimizing sparse matrix-vector multiplication using index and value compression. In Proceedings of the Conference on Computing Frontiers (CF'08). ACM, New York, NY, 87-96.

Langou, J., Langou, J., Luszczek, P., Kurzak, J., Buttari, A., And Dongarra, J. 2006 . Exploiting the performance of 32 bit floating point arithmetic in obtaining 64 bit accuracy (revisiting iterative refinement for linear systems). In Proceedings of the ACM / IEEE Conference on Supercomputing (SC'06). ACM, New York, NY, 113.

Lee, B., Vuduc, R., Demmel, J., ANd Yelick, K. 15-18 Aug. 2004. Performance models for evaluation and automatic tuning of symmetric sparse matrix-vector multiply. In Proceedings of the International Conference on Parallel Processing (ICPP'04). 169-176, vol. 1.

Mellor-Crummey, J. and Garvin, J. 2004. Optimizing sparse matrix-vector product computations using unroll and jam. Int. J. High Perform. Comput. Appl. 18, 2, 225.

Moloney, D., Geraghty, D., McSweeney, C., ANd McElroy, C. 2005. Streaming sparse matrix compression/decompression. In Proceedings of the High Performance Embedded Architectures and Compilers, 1st International Conference (HiPEAC'05). 116-129.

Pichel, J. C., Heras, D. B., Cabaleiro, J. C., ANd Rivera, F. F. 2004 . Improving the locality of the sparse matrix-vector product on shared memory multiprocessors. In Proceedings of the Economics Conference on Parallel, Distributed, and Network-Based Processing. 66.

Pinar, A. AND Heath, M. 1999. Improving performance of sparse matrix-vector multiplication. In Proceedings of the Supercomputing'99 Conference. ACM SIGARCH and IEEE.

SAAD, Y. 1994. SPARSKIT: A basic tool kit for sparse matrix computations. Tech. rep., Computer Science Department, University of Minnesota, Minneapolis, MN.

SAAD, Y. 2003. Iterative Methods for Sparse Linear Systems. SIAM, Philadelphia, PA.

Temam, O. AND Jalby, W. 1992. Characterizing the behavior of sparse algorithms on caches. In Proceedings of the Supercomputing'92 Conference. IEEE, 578-587.

TolEDo, S. 1997. Improving the memory-system performance of sparse-matrix vector multiplication. IBM J. Resear. Devel. 41, 6, 711-725.

Vuduc, R., Demmel, J., Yelick, K., Kamil, S., Nishtala, R., and Lee, B. 2002. Performance optimizations and bounds for sparse matrix-vector multiply. In Proceedings of the Supercomputing Conference'02. 26-26.

Vuduc, R. W. ANd Moon, H. 2005. Fast sparse matrix-vector multiplication by exploiting variable block structure. In Proceedings of the Conference on High Performance Computing and Communications. Lecture Notes in Computer Science, vol. 3726. Springer, 807-816.

White, J. AND SADAYAPPAN, P. 1997. On improving the performance of sparse matrix-vector multiplication. In Proceedings of the 4th International Conference on High Performance Computing (HiPC'97).

ACM Transactions on Architecture and Code Optimization, Vol. 7, No. 3, Article 16, Pub. date: December 2010. 
WiLLCOCK, J. AND LumSDAINE, A. 2006. Accelerating sparse matrix computations via data compression. In Proceedings of the 20th Annual International Conference on Supercomputing (ICS'06). ACM Press, New York, NY, 307-316.

Williams, S., Oilker, L., Vuduc, R., Shalf, J., Yelick, K., and Demmel, J. 2007. Optimization of sparse matrix-vector multiplication on emerging multicore platforms. In Proceedings of the ACM/IEEE Conference on Supercomputing.

Received May 2009; revised February 2010; accepted April 2010 